\title{
ADAPTIVE MESH METHODS FOR ONE- AND TWO-DIMENSIONAL HYPERBOLIC CONSERVATION LAWS*
}

\author{
HUAZHONG TANG ${ }^{\dagger}$ AND TAO TANG
}

\begin{abstract}
We develop efficient moving mesh algorithms for one- and two-dimensional hyperbolic systems of conservation laws. The algorithms are formed by two independent parts: PDE evolution and mesh-redistribution. The first part can be any appropriate high-resolution scheme, and the second part is based on an iterative procedure. In each iteration, meshes are first redistributed by an equidistribution principle, and then on the resulting new grids the underlying numerical solutions are updated by a conservative-interpolation formula proposed in this work. The iteration for the meshredistribution at a given time step is complete when the meshes governed by a nonlinear equation reach the equilibrium state. The main idea of the proposed method is to keep the mass-conservation of the underlying numerical solution at each redistribution step. In one dimension, we can show that the underlying numerical approximation obtained in the mesh-redistribution part satisfies the desired TVD property, which guarantees that the numerical solution at any time level is TVD, provided that the PDE solver in the first part satisfies such a property. Several test problems in one and two dimensions are computed using the proposed moving mesh algorithm. The computations demonstrate that our methods are efficient for solving problems with shock discontinuities, obtaining the same resolution with a much smaller number of grid points than the uniform mesh approach.
\end{abstract}

Key words. adaptive mesh method, hyperbolic conservation laws, finite volume method

AMS subject classifications. 65M93, 35L64, 76N10

PII. S003614290138437X

1. Introduction. Adaptive mesh methods have important applications for a variety of physical and engineering areas such as solid and fluid dynamics, combustion, heat transfer, material science, etc. The physical phenomena in these areas develop dynamically singular or nearly singular solutions in fairly localized regions, such as shock waves, boundary layers, detonation waves, etc. The numerical investigation of these physical problems may require extremely fine meshes over a small portion of the physical domain to resolve the large solution variations. In multidimensions, developing effective and robust adaptive grid methods for these problems becomes necessary. Successful implementation of the adaptive strategy can increase the accuracy of the numerical approximations and also decrease the computational cost. In the past two decades, there has been important progress in developing mesh methods for PDEs, including the variational approach of Winslow [36], Brackbill [5], and Brackbill and Saltzman [6]; finite element methods by Miller and Miller [25] and Davis and Flaherty [11]; the moving mesh PDEs of Cao, Huang, and Russell [7], Stockie, Mackenzie, and Russell [33], Li and Petzold [23], and Ceniceros and Hou [8]; and moving mesh methods based on harmonic mapping of Dvinsky [12] and Li, Tang, and Zhang [21, 22].

${ }^{*}$ Received by the editors February 1, 2001; accepted for publication (in revised form) September 26, 2002; published electronically April 23, 2003. This research was supported by Hong Kong Baptist University and Hong Kong Research Grants Council.

http://www.siam.org/journals/sinum/41-2/38437.html

$\dagger$ Institute of Computational Mathematics, Academy of Mathematics and System Sciences, Chinese Academy of Sciences, P.O. Box 2719, Beijing 100080, People's Republic of China. Current address: School of Mathematical Sciences, Peking University, Beijing 100871, People's Republic of China (hztang@lsec.cc.ac.cn, hztang@math.pku.edu.cn). The research of this author was partially supported by the National Natural Science Foundation of China (19901031) and the Special Funds for Major State Basic Research Projects of China.

${ }^{\ddagger}$ Department of Mathematics, Hong Kong Baptist University, Kowloon Tong, Hong Kong, People’s Republic of China (ttang@math.hkbu.edu.hk). 
Harten and Hyman [14] began the earliest study in this direction, by moving the grid at an adaptive speed in each time step to improve the resolution of shocks and contact discontinuities. After their work, many other moving mesh methods for hyperbolic problems have been proposed in the literature, including those of Azarenok and collaborators [1, 2, 3], Fazio and LeVeque [13], Liu, Ji, and Liao [24], Saleri and Steinberg [29], and Stockie, Mackenzie, and Russell [33]. However, many existing moving mesh methods for hyperbolic problems are designed for one space dimension. In one dimension, it is generally possible to compute on a very fine grid, and so the need for moving mesh methods may not be clear. Multidimensional moving mesh methods are often difficult to use in fluid dynamics problems, since the grid will typically suffer large distortions and possible tangling. It is therefore useful to design a simple and robust moving mesh algorithm for hyperbolic problems in multidimensions.

The main objective of this paper is to develop one- and two-dimensional (1D and 2D) moving mesh methods for hyperbolic systems of conservation laws. Following Li, Tang, and Zhang [21] we propose a moving mesh method containing two separate parts: PDE time-evolution and mesh-redistribution. The first part can be any suitable high-resolution method such as the wave-propagation algorithm, central schemes, and ENO methods. Once numerical solutions are obtained at the given time level, the mesh will be redistributed using an iteration procedure. At each iteration, the grid is moved according to a variational principle, and the underlying numerical solution on the new grid will be updated using some simple methods (such as conventional interpolation). It is noted that the direct use of conventional interpolation is unsatisfactory for hyperbolic problems, since many physical properties such as mass-conservation and TVD (in one dimension) may be destroyed. In order to preserve these physical properties, we propose to use conservative-interpolation in the solution-updating step. The idea of using conservative-interpolation is new and is shown to work successfully for hyperbolic problems. This approach also preserves the total mass of the numerical solutions, and by the well-known Lax-Wendroff theory the numerical solutions converge to the weak solution of the underlying hyperbolic system.

The paper is organized as follows. In section 2 , we briefly review some theory of the variational approach for moving mesh methods, which is relevant to the meshredistribution part of our algorithm. In section 3, we propose a 1D moving mesh algorithm for solving hyperbolic systems of conservation laws, which will be extended to a $2 \mathrm{D}$ algorithm in section 4 . Numerical experiments are carried out in sections 5 and 6 , where several $1 \mathrm{D}$ and $2 \mathrm{D}$ examples are considered.

2. Mesh generation based on the variational approach. Let $\vec{x}=\left(x_{1}, x_{2}, \ldots\right.$, $\left.x_{d}\right)$ and $\vec{\xi}=\left(\xi_{1}, \xi_{2}, \ldots, \xi_{d}\right)$ denote the physical and computational coordinates, respectively. Here $d \geq 1$ denotes the number of spatial dimensions. A one-to-one coordinate transformation from the computational (or logical) domain $\Omega_{c}$ to the physical domain $\Omega_{p}$ is denoted by

$$
\vec{x}=\vec{x}(\vec{\xi}), \quad \vec{\xi} \in \Omega_{c}
$$

Its inversion is denoted by

$$
\vec{\xi}=\vec{\xi}(\vec{x}), \quad \vec{x} \in \Omega_{p}
$$


In the variational approach, the mesh map is provided by the minimizer of a functional of the following form:

$$
E(\vec{\xi})=\frac{1}{2} \sum_{k} \int_{\Omega_{p}} \nabla \xi_{k}^{T} G_{k}^{-1} \nabla \xi_{k} d \vec{x}
$$

where $\nabla:=\left(\partial_{x_{1}}, \partial_{x_{2}}, \ldots, \partial_{x_{d}}\right)^{T}$ and $G_{k}$ are given symmetric positive definite matrices called monitor functions. In general, monitor functions depend on the underlying solution to be adapted. More terms can be added to the functional (2.3) to control other aspects of the mesh such as orthogonality and mesh alignment with a given vector field $[5,6]$.

The variational mesh is determined by the Euler-Lagrange equation of the above functional:

$$
\nabla \cdot\left(G_{k}^{-1} \nabla \xi_{k}\right)=0, \quad 1 \leq k \leq d .
$$

One of the simplest choices of monitor functions is $G_{k}=\omega I, 1 \leq k \leq d$, where $I$ is the identity matrix and $\omega$ is a positive weight function, e.g., $\omega=\sqrt{1+|\nabla u|^{2}}$. Here $u$ is the solution of the underlying PDE. In this case, we obtain Winslow's variable diffusion method [36]:

$$
\nabla \cdot\left(\frac{1}{\omega} \nabla \xi_{k}\right)=0, \quad 1 \leq k \leq d
$$

By using the above equations, a map between the physical domain $\Omega_{p}$ and the logical domain $\Omega_{c}$ can be computed. Typically, the map transforms a uniform mesh in the logical domain, clustering grid points in those regions of the physical domain where the solution has the largest gradients.

2.1. 1D case. Although the main objective of this work is to provide an effective moving mesh algorithm for $2 \mathrm{D}$ conservation laws, it is easier to illustrate the basic moving mesh ideas by starting with some $1 \mathrm{D}$ discussions. Let $x$ and $\xi$ denote the physical and computational coordinates, respectively, which are (without loss of generality) assumed to be in $[a, b]$ and $[0,1]$, respectively. A one-to-one coordinate transformation between these domains is denoted by

$$
\begin{array}{ll}
x=x(\xi), & \xi \in[0,1], \\
x(0)=a, & x(1)=b .
\end{array}
$$

The 1D Euler-Lagrange equation has the form

$$
\left(\omega^{-1} \xi_{x}\right)_{x}=0 .
$$

Using the above equation, we can obtain the conventional 1D equidistribution principle: $\omega x_{\xi}=$ constant, or equivalently,

$$
\left(\omega x_{\xi}\right)_{\xi}=0 .
$$

Both (2.7) and (2.8) have the same form, and therefore solving either of them will yield the desired mesh map $x=x(\xi)$. However, the situation is different in the 2D case, where we will choose to solve equations of the form (2.8), as will be described in the next subsection. 
2.2. 2D case. We will consider the Winslow's variable diffusion method (2.5). The extension to the general Euler-Lagrange equation (2.4) is straightforward. Let $(x, y)=(x(\xi, \eta), y(\xi, \eta))$ be the mesh map in two dimensions. Then (2.5) becomes

$$
\begin{aligned}
& \left(\omega^{-1} \xi_{x}\right)_{x}+\left(\omega^{-1} \xi_{y}\right)_{y}=0, \\
& \left(\omega^{-1} \eta_{x}\right)_{x}+\left(\omega^{-1} \eta_{y}\right)_{y}=0 .
\end{aligned}
$$

In practice, the physical domain $\Omega_{p}$ may have a very complex geometry, and as a result, solving the elliptic system (2.9) directly on structured grids is unrealistic. Therefore we usually solve the corresponding mesh generation equations on the computational domain $\Omega_{c}$ by interchanging the dependent and independent variables in (2.9):

$$
\begin{aligned}
& \frac{x_{\xi}}{J}[\left.\left(x_{\eta} \frac{1}{J \omega} x_{\eta}+y_{\eta} \frac{1}{J \omega} y_{\eta}\right)_{\xi}-\left(x_{\xi} \frac{1}{J \omega} x_{\eta}+y_{\xi} \frac{1}{J \omega} y_{\eta}\right)_{\eta}\right] \\
&+\frac{x_{\eta}}{J}\left[-\left(x_{\eta} \frac{1}{J \omega} x_{\xi}+y_{\eta} \frac{1}{J \omega} y_{\xi}\right)_{\xi}+\left(x_{\xi} \frac{1}{J \omega} x_{\xi}+y_{\xi} \frac{1}{J \omega} y_{\xi}\right)_{\eta}\right]=0, \\
& \frac{y_{\xi}}{J}\left[\left(x_{\eta} \frac{1}{J \omega} x_{\eta}+y_{\eta} \frac{1}{J \omega} y_{\eta}\right)_{\xi}-\left(x_{\xi} \frac{1}{J \omega} x_{\eta}+y_{\xi} \frac{1}{J \omega} y_{\eta}\right)_{\eta}\right] \\
& \quad+\frac{y_{\eta}}{J}\left[-\left(x_{\eta} \frac{1}{J \omega} x_{\xi}+y_{\eta} \frac{1}{J \omega} y_{\xi}\right)_{\xi}+\left(x_{\xi} \frac{1}{J \omega} x_{\xi}+y_{\xi} \frac{1}{J \omega} y_{\xi}\right)_{\eta}\right]=0 .
\end{aligned}
$$

Note that system (2.10) is more complicated than the Euler-Lagrange equation (2.9), which requires more computational effort in obtaining numerical approximations. An alternative approach, as observed by Ceniceros and Hou [8], is to consider a functional defined in the computational domain,

$$
\tilde{E}[x, y]=\frac{1}{2} \int_{\Omega_{c}}\left(\widetilde{\nabla}^{T} x G_{1} \widetilde{\nabla} x+\widetilde{\nabla}^{T} y G_{2} \widetilde{\nabla} y\right) d \xi d \eta,
$$

to replace the conventional functional (2.3), where $G_{k}$ are monitor functions, and $\widetilde{\nabla}=\left(\partial_{\xi}, \partial_{\eta}\right)^{T}$. The corresponding Euler-Lagrange equation is

$$
\begin{aligned}
& \partial_{\xi}\left(G_{1} \partial_{\xi} x\right)+\partial_{\eta}\left(G_{1} \partial_{\eta} x\right)=0, \\
& \partial_{\xi}\left(G_{2} \partial_{\xi} y\right)+\partial_{\eta}\left(G_{2} \partial_{\eta} y\right)=0 .
\end{aligned}
$$

In particular, with the choice $G=\omega I$ we have

$$
\widetilde{\nabla} \cdot(\omega \widetilde{\nabla} x)=0, \quad \widetilde{\nabla} \cdot(\omega \widetilde{\nabla} y)=0 .
$$

The monitor functions will be chosen based on the properties of the physical solutions.

A typical choice used in $[8]$ is $\omega=\sqrt{1+\alpha_{1}|u|^{2}+\alpha_{2}|\widetilde{\nabla} u|^{2}}$ or $\omega=\sqrt{1+\alpha_{1}|u|^{2}+\alpha_{2}|\nabla u|^{2}}$, where $\alpha_{1}, \alpha_{2}$ are some nonnegative constants.

3. 1D algorithm. For convenience, we assume that a fixed uniform mesh on the computational domain is given by $\xi_{j}=j /(J+1), 0 \leq j \leq J+1$. We denote the cell average of the solution $u(x)$ over the interval $\left[x_{j}, x_{j+1}\right]$ as

$$
u_{j+\frac{1}{2}}=\frac{1}{\Delta x_{j+\frac{1}{2}}} \int_{x_{j}}^{x_{j+1}} u(x) d x
$$


where $\Delta x_{j+\frac{1}{2}}=x_{j+1}-x_{j}$. In practice, the monitor function $\omega$ is always associated with the underlying solution $u$ or/and its derivatives, but without loss of generality we assume that $\omega=\omega(u)$. For monitor functions involving first or second derivatives, central differencing will be used to approximate these derivatives.

3.1. Mesh-redistribution. In order to solve the mesh-redistribution equation (2.8), we introduce an artificial time $\tau$ and solve

$$
x_{\tau}=\left(\omega x_{\xi}\right)_{\xi}, \quad 0<\xi<1,
$$

subject to boundary conditions $x(0, \tau)=a$ and $x(1, \tau)=b$. We discretize (3.1) on the uniform mesh in $\Omega_{c}$ :

$$
\tilde{x}_{j}=x_{j}+\frac{\Delta \tau}{\Delta \xi^{2}}\left[\omega\left(u_{j+\frac{1}{2}}\right)\left(x_{j+1}-x_{j}\right)-\omega\left(u_{j-\frac{1}{2}}\right)\left(x_{j}-x_{j-1}\right)\right], \quad 1 \leq j \leq J,
$$

where $\Delta \xi=1 /(J+1)$ is the step size in $\Omega_{c}$. Solving (3.2) with boundary conditions $x_{0}=a$ and $x_{J+1}=b$ leads to a new grid in the physical domain $\Omega_{p}$. Some advantages of using the approach (3.1) and (3.2) to solve the mesh redistribution equation (2.8) will be seen from Lemma 3.1 and Theorem 3.1.

3.2. Solution-updating on new grids. After obtaining the new grid $\left\{\widetilde{x}_{j}\right\}$, we need to update $u$ at the grid point $\widetilde{x}_{j+\frac{1}{2}}=\left(\widetilde{x}_{j}+\widetilde{x}_{j+1}\right) / 2$ based on the knowledge of $\left\{x_{j+\frac{1}{2}}, \widetilde{x}_{j+\frac{1}{2}}, u_{j+\frac{1}{2}}\right\}$. The traditional way to do this is using the conventional interpolation

$$
\widetilde{u}_{j+\frac{1}{2}}=u_{k+\frac{1}{2}}+\frac{u_{k+\frac{1}{2}}-u_{k-\frac{1}{2}}}{x_{k+\frac{1}{2}}-x_{k-\frac{1}{2}}}\left(\widetilde{x}_{j+\frac{1}{2}}-x_{k+\frac{1}{2}}\right) \quad \text { if } \widetilde{x}_{j+\frac{1}{2}} \in\left[x_{k-\frac{1}{2}}, x_{k+\frac{1}{2}}\right] .
$$

Since the monitor function $\omega$ is dependent on the underlying solution $u$, the grid redistribution equations (3.2)-(3.3) form a nonlinear system. It is therefore natural to make several iterations to solve (3.2)-(3.3) in order to gain better control of the grid distribution near those regions where the solution $u$ has a large gradient. In solving hyperbolic conservation laws with strong discontinuities (e.g., shocks), iteration techniques based on (3.2)-(3.3) have been employed, and it is found that the results for the solution and the mesh are not satisfactory. The main problem is that the linear interpolation (3.3) cannot preserve conservation of mass, which, by the Lax-Wendroff theory, is an essential requirement for a good numerical scheme for hyperbolic conservation laws.

In the following we will introduce a new method to update $u$, noting that massconservation is an essential requirement for hyperbolic conservation laws. To begin with, assume that the difference between $\tilde{x}_{j+\frac{1}{2}}$ and $x_{j+\frac{1}{2}}$ is small. Let $\widetilde{u}_{j+\frac{1}{2}}$ and $u_{j+\frac{1}{2}}$ be cell averages of the solution $u(x)$ over the intervals $\left[\widetilde{x}_{j}, \widetilde{x}_{j+1}\right]$ and $\left[x_{j}, x_{j+1}\right]$, respectively. We will derive a formula for $\widetilde{u}_{j+\frac{1}{2}}$ using the perturbation method. If 
$\widetilde{x}=x-c(x)$ with a small displacement $c(x)$, i.e., $|c(x)| \ll 1$, then we have

$$
\begin{aligned}
\int_{\widetilde{x}_{j}}^{\widetilde{x}_{j+1}} \tilde{u}(\widetilde{x}) d \widetilde{x} & =\int_{x_{j}}^{x_{j+1}} u(x-c(x))\left(1-c^{\prime}(x)\right) d x \\
& \approx \int_{x_{j}}^{x_{j+1}}\left(u(x)-c(x) u_{x}(x)\right)\left(1-c^{\prime}(x)\right) d x \\
& \approx \int_{x_{j}}^{x_{j+1}}\left(u(x)-(c u)_{x}\right) d x \\
& =\int_{x_{j}}^{x_{j+1}} u(x) d x-\left((c u)_{j+1}-(c u)_{j}\right),
\end{aligned}
$$

where we have neglected higher-order terms, and $(c u)_{j}$ denotes the value of $c u$ at the $j$ th cell interface. The following almost conservative-interpolation formula follows from (3.4):

$$
\Delta \widetilde{x}_{j+\frac{1}{2}} \widetilde{u}_{j+\frac{1}{2}}=\Delta x_{j+\frac{1}{2}} u_{j+\frac{1}{2}}-\left((c u)_{j+1}-(c u)_{j}\right),
$$

where $\Delta \widetilde{x}_{j+\frac{1}{2}}=\widetilde{x}_{j+1}-\widetilde{x}_{j}$ and $c_{j}=x_{j}-\widetilde{x}_{j}$. Note that the above solution-updating method guarantees the conservation of mass in the following sense:

$$
\sum_{j} \Delta \widetilde{x}_{j+\frac{1}{2}} \widetilde{u}_{j+\frac{1}{2}}=\sum_{j} \Delta x_{j+\frac{1}{2}} u_{j+\frac{1}{2}} .
$$

The linear flux $\mathrm{cu}$ in (3.5) will be approximated by some upwinding numerical flux; see (3.11) below.

If the function $u$ is suitably smooth, then it can be shown that the size of the moving speed $c(x)$ is small. It is known that the first and second derivatives of the parabolic-type equation (3.1) are bounded, provided that the initial data and the monitor function satisfy some regularity requirements. By the definition of $c(x)$, we have

$$
\begin{aligned}
& c(x)=x-\widetilde{x}=-\left(x_{\tau}\right) \Delta \tau=\mathcal{O}(\Delta \tau), \\
& c^{\prime}(x)=1-\tilde{x}_{x}=1-\frac{\tilde{x}_{\xi}}{x_{\xi}}=-\frac{x_{\xi \tau}}{x_{\xi}} \Delta \tau=\mathcal{O}(\Delta \tau),
\end{aligned}
$$

which indicate that the moving speed in each cell is indeed very small.

3.3. Solution procedure. Our solution procedure is based on two independent parts: a mesh-redistribution algorithm and a solution algorithm. The first part will be based on an iteration procedure using (3.2) and (3.5). The second part will be independent of the first, and it can be any of the standard codes for solving the given PDEs, such as ENO schemes [31, 39], central schemes [17, 27], relaxation schemes $[16,26,34]$, BGK schemes [38, 35], and several other types of high-resolution methods (see, e.g., $[20,15,18])$. The solution procedure can be illustrated by the following flowchart.

Algorithm 0.

Step 1. Given a uniform (fixed) partition of the logical domain $\Omega_{c}$, use the equidistribution principle (2.8) to generate an initial partition $x_{j}^{[0]}:=x_{j}$ of the physical domain $\Omega_{p}$. Then compute the grid values $u_{j+\frac{1}{2}}^{[0]}$ based on the cell average for the initial data $u(x, 0)$. 
Step 2. Move grid $\left\{x_{j}^{[\nu]}\right\}$ to $\left\{x_{j}^{[\nu+1]}\right\}$ based on scheme (3.2), and compute $\left\{u_{j+\frac{1}{2}}^{[\nu+1]}\right\}$ on the new grid based on scheme (3.5) for $\nu \geq 0$. Repeat the updating procedure for a fixed number of iterations or until $\left\|x^{[\nu+1]}-x^{[\nu]}\right\| \leq \epsilon$. The mesh-redistribution scheme (3.2) can be also replaced by the Gauss-Seidel iteration procedure (3.32), as discussed at the end of this section.

Step 3. Evolve the underlying PDEs using a high-resolution finite volume method on the mesh $\left\{x_{j}^{[\nu+1]}\right\}$ to obtain the numerical approximations $u_{j+\frac{1}{2}}^{n+1}$ at the time level $t_{n+1}$.

Step 4. If $t_{n+1} \leq T$, then let $u_{j+\frac{1}{2}}^{[0]}:=u_{j+\frac{1}{2}}^{n+1}$ and $x_{j}^{[0]}:=x_{j}^{[\nu+1]}$ and go to Step 2.

3.3.1. Some discussions on Step 2. A new mesh $x_{j}^{[\nu+1]}$ is obtained using where

$$
x_{j}^{[\nu+1]}=\alpha_{j+\frac{1}{2}} x_{j+1}^{[\nu]}+\left(1-\alpha_{j+\frac{1}{2}}-\alpha_{j-\frac{1}{2}}\right) x_{j}^{[\nu]}+\alpha_{j-\frac{1}{2}} x_{j-1}^{[\nu]}
$$

$$
\alpha_{j+\frac{1}{2}}=\frac{\Delta \tau}{\Delta \xi^{2}} \omega\left(u_{j+\frac{1}{2}}^{[\nu]}\right) .
$$

The above equation is solved subject to the following stability condition:

$$
\max _{j} \alpha_{j+\frac{1}{2}} \leq \frac{1}{2}
$$

Next, numerical solutions are updated on the new grids $\left\{x_{j}^{[\nu+1]}\right\}$ (at the same time level) using (3.5),

$$
u_{j+\frac{1}{2}}^{[\nu+1]}=\beta_{j}^{[\nu]} u_{j+\frac{1}{2}}^{[\nu]}-\gamma_{j}^{[\nu]}\left((\widehat{c u})_{j+1}^{[\nu]}-(\widehat{c u})_{j}^{[\nu]}\right),
$$

where

$$
\gamma_{j}^{[\nu]}=\left(x_{j+1}^{[\nu+1]}-x_{j}^{[\nu+1]}\right)^{-1}, \quad \beta_{j}^{[\nu]}=\gamma_{j}^{[\nu]} \cdot\left(x_{j+1}^{[\nu]}-x_{j}^{[\nu]}\right),
$$

and the numerical flux $\widehat{c u}_{j}$ is defined by

$$
(\widehat{c u})_{j}=\frac{c_{j}}{2}\left(u_{j+\frac{1}{2}}+u_{j-\frac{1}{2}}\right)-\frac{\left|c_{j}\right|}{2}\left(u_{j+\frac{1}{2}}-u_{j-\frac{1}{2}}\right) .
$$

The wave speed $c_{j}$ above is defined by $c_{j}^{[\nu]}=x_{j}^{[\nu]}-x_{j}^{[\nu+1]}$.

Remark 3.1. In our numerical computation, the first-order numerical flux $(\widehat{c u})_{j}$ defined by (3.11) will be replaced by a second-order one as the following:

$$
(\widehat{c u})_{j}=\frac{c_{j}}{2}\left(u_{j}^{+}+u_{j}^{-}\right)-\frac{\left|c_{j}\right|}{2}\left(u_{j}^{+}-u_{j}^{-}\right),
$$

where $u_{j}^{+}$and $u_{j}^{-}$will be defined by (3.19) below.

Remark 3.2. In practice, it is common to use some temporal or spatial smoothing on the monitor function to obtain smoother meshes. One of the reasons for using smoothing is to avoid very singular mesh and/or large approximation errors near those regions where the solution has a large gradient. In this work, we apply the following low pass filter to smooth the monitor:

$$
\omega_{j+\frac{1}{2}} \leftarrow \frac{1}{4}\left(\omega_{j+\frac{3}{2}}+2 \omega_{j+\frac{1}{2}}+\omega_{j-\frac{1}{2}}\right),
$$

where $\omega_{j+\frac{1}{2}}=\omega\left(u_{j+\frac{1}{2}}\right)$. 
3.3.2. Some discussions on Step 3. This step is independent of Step 2, and, as a result, it can be done using any efficient modern numerical technique for hyperbolic conservation laws. As an example, we consider a second-order finite volume approach to solving the 1D scalar hyperbolic conservation laws

$$
u_{t}+f(u)_{x}=0, \quad t>0,
$$

with compactly supported initial condition

$$
u(x, 0)=u_{0}(x), \quad u_{0} \in L^{\infty} \cap B V .
$$

Integrating (3.14) over the control volume $\left[t_{n}, t_{n+1}\right) \times\left[x_{j}, x_{j+1}\right]$ leads to the following (explicit) finite volume method:

$$
u_{j+\frac{1}{2}}^{n+1}=u_{j+\frac{1}{2}}^{n}-\frac{t_{n+1}-t_{n}}{x_{j+1}-x_{j}}\left(\widehat{f}_{j+1}^{n}-\widehat{f}_{j}^{n}\right),
$$

where $\widehat{f}_{j}^{n}$ is some appropriate numerical flux satisfying

$$
\widehat{f}_{j}^{n}=\widehat{f}\left(u_{j}^{n,-}, u_{j}^{n,+}\right), \quad \widehat{f}(u, u)=f(u) .
$$

An example of such a numerical flux is the Lax-Friedrichs flux:

$$
\widehat{f}(a, b)=\frac{1}{2}\left[f(a)+f(b)-\max _{u}\left\{\left|f_{u}\right|\right\}(b-a)\right] .
$$

In (3.17), $u_{j}^{n, \pm}$ are defined by

$$
u_{j}^{n, \pm}=u_{j \pm \frac{1}{2}}^{n}+\frac{1}{2}\left(x_{j}-x_{j \pm 1}\right) \tilde{S}_{j \pm \frac{1}{2}},
$$

where $\tilde{S}_{j+\frac{1}{2}}$ is an approximation of the slope $u_{x}$ at $x_{j+\frac{1}{2}}$, defined by

$$
\tilde{S}_{j+\frac{1}{2}}=\left(\operatorname{sign}\left(\tilde{S}_{j+\frac{1}{2}}^{+}\right)+\operatorname{sign}\left(\tilde{S}_{j+\frac{1}{2}}^{-}\right)\right) \frac{\left|\tilde{S}_{j+\frac{1}{2}}^{+} \tilde{S}_{j+\frac{1}{2}}^{-}\right|}{\left|\tilde{S}_{j+\frac{1}{2}}^{+}\right|+\left|\tilde{S}_{j+\frac{1}{2}}^{-}\right|},
$$

with

$$
\tilde{S}_{j+\frac{1}{2}}^{+}=\frac{u_{j+\frac{3}{2}}^{n}-u_{j+\frac{1}{2}}^{n}}{x_{j+\frac{3}{2}}-x_{j+\frac{1}{2}}}, \quad \tilde{S}_{j+\frac{1}{2}}^{-}=\frac{u_{j+\frac{1}{2}}^{n}-u_{j-\frac{1}{2}}^{n}}{x_{j+\frac{1}{2}}-x_{j-\frac{1}{2}}} .
$$

The MUSCL (monotone upstream-centered scheme for conservation laws)-type finite volume method (3.16)-(3.18), which is of second-order accuracy in smooth regions, will be applied in the 1D numerical experiments.

3.4. Some theoretical results on the adaptive mesh solutions. In one dimension, some good theoretical guarantees for the numerical grids can be obtained. In the following, we prove some theoretical results for the mesh-redistribution equation (3.7) and the solution-updating equation (3.9). We first demonstrate that the new mesh $x^{[\nu+1]}$ generated by (3.7) keeps the monotonic order of $x^{[\nu]}$.

Lemma 3.1. Assume $x_{j+1}^{[\nu]}>x_{j}^{[\nu]}$ for $0 \leq j \leq J$. If the new mesh $x^{[\nu+1]}$ is obtained using (3.7), with $\alpha_{j+\frac{1}{2}}$ satisfying the stability condition (3.8), then $x_{j-1}^{[\nu+1]}<$ $x_{j}^{[\nu]}<x_{j+1}^{[\nu+1]}$ for $1 \leq j \leq J$, and $x_{j+1}^{[\nu+1]}>x_{j}^{[\nu+1]}$ for $0 \leq j \leq J$. 
Proof. Using the stability condition (3.8) gives $1-\alpha_{j+\frac{1}{2}}-\alpha_{j-\frac{1}{2}} \geq 0$. Moreover, $\alpha_{j \pm \frac{1}{2}}$ are all positive. Therefore, it follows from (3.7) and the assumption $x_{j+1}^{[\nu]}>x_{j}^{[\nu]}$ that $x_{j-1}^{[\nu+1]}<x_{j}^{[\nu]}<x_{j+1}^{[\nu+1]}$. We now rewrite (3.7) into the following form:

$$
x_{j}^{[\nu+1]}=\alpha_{j+\frac{1}{2}} \Delta x_{j+\frac{1}{2}}^{[\nu]}+x_{j}^{[\nu]}-\alpha_{j-\frac{1}{2}} \Delta x_{j-\frac{1}{2}}^{[\nu]},
$$

where $\Delta x_{j+\frac{1}{2}}=x_{j+1}-x_{j}$. It follows from the above equation that

$$
\Delta x_{j-\frac{1}{2}}^{[\nu+1]}=\alpha_{j+\frac{1}{2}} \Delta x_{j+\frac{1}{2}}^{[\nu]}+\left(1-2 \alpha_{j-\frac{1}{2}}\right) \Delta x_{j-\frac{1}{2}}^{[\nu]}+\alpha_{j-\frac{3}{2}} \Delta x_{j-\frac{3}{2}}^{[\nu]} .
$$

Since the first and last coefficients of the right-hand side are positive and the second one is nonnegative (due to the stability condition (3.8)), the assumption $x_{j+1}^{[\nu]}>x_{j}^{[\nu]}$ yields $\Delta x_{j-\frac{1}{2}}^{[\nu+1]}>0$. This shows that $x_{j+1}^{[\nu+1]}>x_{j}^{[\nu+1]}$ for $0 \leq j \leq J$.

Remark 3.3. A consequence of Lemma 3.1 is that $x_{j}^{[\nu+1]} \in\left(x_{j-1}^{[\nu]}, x_{j+1}^{[\nu]}\right)$, which implies that the speed of mesh moving is finite. This is important in better controlling grid distribution near the regions of large gradients in the solution.

Next, we provide a necessary condition under which the updated solution $u_{j+\frac{1}{2}}^{[\nu+1]}$ satisfies the TVD property.

LEMma 3.2. Assume that the initial data $u^{[0]}$ is compactly supported and that the stability condition (3.8) is satisfied. If $x_{j-1}^{[\nu+1]} \leq x_{j}^{[\nu]} \leq x_{j+1}^{[\nu+1]}$ and $x_{j+1}^{[\nu+1]}>x_{j}^{[\nu+1]}$, then the solution-updating scheme (3.9)-(3.11) satisfies

$$
\operatorname{TV}\left(u^{[\nu+1]}\right) \leq \operatorname{TV}\left(u^{[\nu]}\right),
$$

where the total variation is defined by

$$
\operatorname{TV}(u):=\sum_{j}\left|u_{j+\frac{1}{2}}-u_{j-\frac{1}{2}}\right| .
$$

Proof. For ease of notation we denote $\tilde{x}=x^{[\nu+1]}, x=x^{[\nu]}, \tilde{u}=u^{[\nu+1]}, u=u^{[\nu]}$. Note that $c_{j+1}-c_{j}=\Delta x_{j+\frac{1}{2}}-\Delta \tilde{x}_{j+\frac{1}{2}}$. This fact, together with the scheme (3.9) and the numerical flux (3.11), gives

$$
\begin{aligned}
\Delta \tilde{x}_{j+\frac{1}{2}} \tilde{u}_{j+\frac{1}{2}}=\left(c_{j+1}-c_{j}+\Delta \tilde{x}_{j+\frac{1}{2}}\right) u_{j+\frac{1}{2}}+\frac{1}{2}\left(\left|c_{j+1}\right|-c_{j+1}\right) u_{j+\frac{3}{2}} \\
\quad+\frac{1}{2}\left(c_{j}-\left|c_{j}\right|-c_{j+1}-\left|c_{j+1}\right|\right) u_{j+\frac{1}{2}}+\frac{1}{2}\left(\left|c_{j}\right|+c_{j}\right) u_{j-\frac{1}{2}} \\
=\Delta \tilde{x}_{j+\frac{1}{2}} u_{j+\frac{1}{2}}+m_{j+1} u_{j+\frac{3}{2}}-m_{j+1} u_{j+\frac{1}{2}}-M_{j} u_{j+\frac{1}{2}}+M_{j} u_{j-\frac{1}{2}}
\end{aligned}
$$

where $M_{j}=\max \left(0, c_{j}\right)$ and $m_{j}=-\min \left(0, c_{j}\right)$. Note that both $M_{j}$ and $m_{j}$ are nonnegative. It follows from the above result that

$$
\tilde{u}_{j+\frac{1}{2}}=u_{j+\frac{1}{2}}+\frac{m_{j+1}}{\Delta \tilde{x}_{j+\frac{1}{2}}} \Delta u_{j+1}-\frac{M_{j}}{\Delta \tilde{x}_{j+\frac{1}{2}}} \Delta u_{j},
$$

where $\Delta u_{j}=u_{j+\frac{1}{2}}-u_{j-\frac{1}{2}}$. It follows from (3.22) that

$$
\Delta \tilde{u}_{j}=\Delta u_{j}+\frac{m_{j+1}}{\Delta \tilde{x}_{j+\frac{1}{2}}} \Delta u_{j+1}-\left(\frac{M_{j}}{\Delta \tilde{x}_{j+\frac{1}{2}}}+\frac{m_{j}}{\Delta \tilde{x}_{j-\frac{1}{2}}}\right) \Delta u_{j}+\frac{M_{j-1}}{\Delta \tilde{x}_{j-\frac{1}{2}}} \Delta u_{j-1},
$$


which gives

$$
\sum_{j}\left|\Delta \tilde{u}_{j}\right| \leq \sum_{j} \frac{m_{j}}{\Delta \tilde{x}_{j-\frac{1}{2}}}\left|\Delta u_{j}\right|+\sum_{j}\left|1-\frac{M_{j}}{\Delta \tilde{x}_{j+\frac{1}{2}}}-\frac{m_{j}}{\Delta \tilde{x}_{j-\frac{1}{2}}}\right|\left|\Delta u_{j}\right| \sum_{j} \frac{M_{j}}{\Delta \tilde{x}_{j+\frac{1}{2}}}\left|\Delta u_{j}\right| .
$$

It can be verified using the definition of $c_{j}$ that the condition $\tilde{x}_{j-1} \leq x_{j} \leq \tilde{x}_{j+1}$ is equivalent to $-\Delta \tilde{x}_{j-\frac{1}{2}} \leq c_{j} \leq \Delta \tilde{x}_{j+\frac{1}{2}}$. This fact, together with the observation that $M_{j}=0$ when $c_{j} \leq 0$ and $m_{j}=0$ when $c_{j} \geq 0$, yields

$$
1-\frac{M_{j}}{\Delta \tilde{x}_{j+\frac{1}{2}}}-\frac{m_{j}}{\Delta \tilde{x}_{j-\frac{1}{2}}} \geq 0
$$

It follows from (3.23) and (3.24) that $\operatorname{TV}(\tilde{u}) \leq \mathrm{TV}(u)$.

With the two ingredients above, the following TVD property for Step 2 of Algorithm 0 is established.

THEOREM 3.1. Assume that the initial data $u^{[0]}$ is compactly supported and that the stability condition (3.8) is satisfied. Then the iterated mesh and solution $\left\{x^{[\nu+1]}, u^{[\nu+1]}\right\}$ generated by (3.7)-(3.11) satisfies $\operatorname{TV}\left(u^{[\nu+1]}\right) \leq \operatorname{TV}\left(u^{[\nu]}\right)$.

Remark 3.4. If the PDE solver in Step 3 of Algorithm 0 is TVB (i.e., TVbounded) (or TVD), then the above theorem guarantees the TVB (or TVD) property of the moving mesh solution at any time level. It can be also proved similarly that the $l^{\infty}$ - and the $l^{1}$-stabilities are also preserved.

THEOREM 3.2. Assume that the initial function $u_{0}$ in (3.15) is compactly supported and that the stability condition (3.8) is satisfied. Then the moving mesh solution generated by Algorithm 0, with (3.7)-(3.11) for Step 2 and (3.16) for Step 3, is a weak solution of the conservation law (3.14).

Proof. Without loss of generality we assume that only one iteration is used in Step 2 of Algorithm 0. Then, given $\left\{x_{j}^{n}, u_{j+\frac{1}{2}}^{n}\right\}$, the solution $\left\{x_{j}^{n+1}, u_{j+\frac{1}{2}}^{n+1}\right\}$ is computed by the following operator-splitting-type algorithm:

$$
\begin{aligned}
& U_{j+\frac{1}{2}}^{n}=u_{j+\frac{1}{2}}^{n}-\frac{\Delta t_{n}}{\Delta x_{j+\frac{1}{2}}^{n}}\left(f_{j+1}^{n}-f_{j}^{n}\right), \\
& x_{j}^{n+1}=x_{j}^{n}+\frac{\Delta \tau}{\Delta \xi^{2}}\left[\omega\left(U_{j+\frac{1}{2}}^{n}\right)\left(x_{j+1}^{n}-x_{j}^{n}\right)-\omega\left(U_{j-\frac{1}{2}}^{n}\right)\left(x_{j}^{n}-x_{j-1}^{n}\right)\right], \\
& \Delta x_{j+\frac{1}{2}}^{n+1} u_{j+\frac{1}{2}}^{n+1}=\Delta x_{j+\frac{1}{2}}^{n} U_{j+\frac{1}{2}}^{n}-\left(\left(c U^{n}\right)_{j+1}-\left(c U^{n}\right)_{j}\right),
\end{aligned}
$$

where $c_{j}=x_{j}^{n}-x_{j}^{n+1}$ and the numerical flux $f_{j}^{n}$ satisfies the consistency requirement. Multiplying the first equation above by a test function $\phi \in C_{0}^{\infty}(\mathbf{R} \times(0, T])$ gives

$$
\Delta x_{j+\frac{1}{2}}^{n} U_{j+\frac{1}{2}}^{n} \phi\left(x_{j}^{n}, t_{n}\right)=\Delta x_{j+\frac{1}{2}}^{n} u_{j+\frac{1}{2}}^{n} \phi\left(x_{j}^{n}, t_{n}\right)-\Delta t_{n}\left(f_{j+1}^{n}-f_{j}^{n}\right) \phi\left(x_{j}^{n}, t_{n}\right),
$$

which, together with the interpolation step (3.27), gives

$$
\begin{aligned}
& \Delta x_{j+\frac{1}{2}}^{n+1} u_{j+\frac{1}{2}}^{n+1} \phi\left(x_{j}^{n}, t_{n}\right)+\left(\left(c U^{n}\right)_{j+1}-\left(c U^{n}\right)_{j}\right) \phi\left(x_{j}^{n}, t_{n}\right) \\
& \quad=\Delta x_{j+\frac{1}{2}}^{n} u_{j+\frac{1}{2}}^{n} \phi\left(x_{j}^{n}, t_{n}\right)-\Delta t_{n}\left(f_{j+1}^{n}-f_{j}^{n}\right) \phi\left(x_{j}^{n}, t_{n}\right) .
\end{aligned}
$$


Standard summation by parts yields

$$
\begin{aligned}
& \sum_{j} \sum_{n=0}^{N}\left[\Delta x_{j+\frac{1}{2}}^{n+1} u_{j+\frac{1}{2}}^{n+1}-\Delta x_{j+\frac{1}{2}}^{n} u_{j+\frac{1}{2}}^{n}\right] \phi\left(x_{j}^{n}, t_{n}\right) \\
& \quad=-\sum_{j} \sum_{n=0}^{N}\left(\left(c U^{n}\right)_{j+1}-\left(c U^{n}\right)_{j}\right) \phi\left(x_{j}^{n}, t_{n}\right)-\sum_{n=0}^{N} \sum_{j} \Delta t_{n}\left(f_{j+1}^{n}-f_{j}^{n}\right) \phi\left(x_{j}^{n}, t_{n}\right)
\end{aligned}
$$

and then

$$
\begin{aligned}
& -\sum_{j} \Delta x_{j+\frac{1}{2}}^{0} u_{j+\frac{1}{2}}^{0} \phi\left(x_{j}^{0}, 0\right)-\sum_{j} \sum_{n=1}^{N}\left[\phi\left(x_{j}^{n}, t_{n}\right)-\phi\left(x_{j}^{n-1}, t_{n-1}\right)\right] \Delta x_{j+\frac{1}{2}}^{n} u_{j+\frac{1}{2}}^{n} \\
& =\sum_{j} \sum_{n=0}^{N}\left[\phi\left(x_{j}^{n}, t_{n}\right)-\phi\left(x_{j-1}^{n}, t_{n}\right)\right]\left(x_{j}^{n}-x_{j}^{n+1}\right) U_{j}^{n} \\
& \quad+\sum_{n=0}^{N} \sum_{j} \Delta t_{n}\left[\phi\left(x_{j}^{n}, t_{n}\right)-\phi\left(x_{j-1}^{n}, t_{n}\right)\right] f_{j}^{n},
\end{aligned}
$$

where we have used the fact $\phi\left(x, t_{N}\right)=0$ with $t_{N}=T$. We can show that $\Delta x_{j+\frac{1}{2}}^{0} \rightarrow 0$ as $J \rightarrow \infty$. Without loss of generality, assume that the monitor function is the one associated with the equidistribution principle, i.e., $\omega(u)=\sqrt{1+u_{x}^{2}}$. Then

$$
L=\sqrt{1+u_{x}^{2}\left(x_{j}^{0}, 0\right)} \Delta x_{j+\frac{1}{2}}^{0}, \quad 0 \leq j \leq J-1,
$$

is a constant independent of $j$. It follows from the definition of $L$ that

$$
L \leq\left(1+\left|u_{x}\left(x_{j}^{0}, 0\right)\right|\right) \Delta x_{j+\frac{1}{2}}^{0}, \quad 0 \leq j \leq J-1,
$$

which gives

$$
J L \leq \text { the size of } u_{0} \text { 's support }+\operatorname{TV}\left(u^{0}\right) .
$$

This, together with the definition (3.30), leads to

$$
\Delta x_{j+\frac{1}{2}}^{0} \leq \text { const. } J^{-1} \rightarrow 0 \quad \text { as } \quad J \rightarrow \infty .
$$

Moreover, since $\left\{x_{j}^{n}\right\}$, with $n \geq 1$, are obtained by solving a parabolic equation, we have $\Delta x_{j+\frac{1}{2}}^{n} \sim \mathcal{O}(\Delta \xi) \rightarrow 0$ for $n \geq 1$. Taking limits on both sides of (3.29) leads to

$-\int u(x, 0) \phi(x, 0) d x-\iint\left(\phi_{x} x_{t}+\phi_{t}\right) u d x d t=-\iint \phi_{x} x_{t} u d x d t+\iint \phi_{x} f(u) d x d t$,

provided that the numerical solution is convergent to $u$ almost everywhere, where for the second term on the LHS (left-hand side) of (3.29) we have used the fact $\phi(x, t)_{t}=\phi_{t} x_{t}+\phi_{t}$, and for the first term on the RHS (right-hand side) we have used the fact that $c_{j}=x_{j}^{n}-x_{j}^{n} \sim x_{t} d t$. The above result leads to

$$
\iint\left(\phi_{t} u+\phi_{x} f(u)\right) d x d t+\int u(x, 0) \phi(x, 0) d x=0,
$$

which indicates that the moving mesh solution is indeed a weak solution of the underlying conservation law. 
3.5. Grid-motion with Gauss-Seidel iteration. In practice, we also use the following Gauss-Seidel-type iteration to solve the mesh moving equation (2.8):

$$
\omega\left(u_{j+\frac{1}{2}}^{[\nu]}\right)\left(x_{j+1}^{[\nu]}-x_{j}^{[\nu+1]}\right)-\omega\left(u_{j-\frac{1}{2}}^{[\nu]}\right)\left(x_{j}^{[\nu+1]}-x_{j-1}^{[\nu+1]}\right)=0 .
$$

It can also be demonstrated that the new mesh $x^{[\nu+1]}$ generated by (3.32) keeps the monotonic order of $x^{[\nu]}$.

Lemma 3.3. Assume $x_{j+1}^{[\nu]}>x_{j}^{[\nu]}$ for $0 \leq j \leq J$. If the new mesh $x^{[\nu+1]}$ is obtained by using the Gauss-Seidel iterative scheme (3.32), with positive monitor function $\omega$, then $x_{j}^{[\nu+1]}>x_{j-1}^{[\nu+1]}$ for $1 \leq j \leq J+1$. Moreover, $x_{j}^{[\nu]}>x_{j-1}^{[\nu+1]}$ for $1 \leq j \leq J+1$.

Proof. We again denote $\tilde{x}=x^{[\nu+1]}, x=x^{[\nu]}$. It follows from (3.32) that

$$
-\alpha_{j} x_{j+1}+\tilde{x}_{j}-\beta_{j} \tilde{x}_{j-1}=0,
$$

where $\alpha_{j}>0, \beta_{j}>0$ (due to the positivity assumption of the monitor function), and $\alpha_{j}+\beta_{j}=1$. It follows from the above equation that

$$
\tilde{x}_{j}-x_{j+1}-\beta_{j}\left(\tilde{x}_{j-1}-x_{j}\right)=\beta_{j}\left(x_{j}-x_{j+1}\right) \leq 0,
$$

which gives that

$$
\tilde{x}_{j}-x_{j+1} \leq\left(\prod_{k=1}^{j} \beta_{k}\right)\left(\tilde{x}_{0}-x_{1}\right)=\left(\prod_{k=1}^{j} \beta_{k}\right)\left(x_{0}-x_{1}\right)<0 .
$$

The above result yields $\tilde{x}_{j}<x_{j+1}$, which, together with (3.32), also leads to $\tilde{x}_{j}>$ $\tilde{x}_{j-1}$.

If we can further show that $x_{j}^{[\nu]} \leq x_{j+1}^{[\nu+1]}$ for the Gauss-Seidel iteration (3.32), then based on Lemma 3.2 the solution-updating scheme (3.9) together with the meshredistribution scheme (3.32) will also satisfy the TVD property, i.e., $\operatorname{TV}\left(u^{[\nu+1]}\right) \leq$ $\operatorname{TV}\left(u^{[\nu]}\right)$. However, it seems unlikely that $x_{j}^{[\nu]} \leq x_{j+1}^{[\nu+1]}$ holds for (3.32) in general situations. On the other hand, the combination of (3.9) and (3.32) has been employed in our numerical experiments, and the numerical results are quite satisfactory. Therefore, both (3.7) and (3.32) can be used in Step 2 of Algorithm 0 to redistribute grid points. In most test problems considered in this work, the grid updating procedure at each time step takes five full Gauss-Seidel iterations, although the difference between solutions with three and five iterations is very small.

4. 2D algorithm. One of the advantages of the adaptive mesh methods described in the last section is that they can be naturally extended to two dimensions. In the following, we briefly discuss this extension, together with the boundary point redistribution technique which is necessary for $2 \mathrm{D}$ mesh redistribution.

4.1. A conservative solution-updating method. In two dimensions, the logical domain $\bar{\Omega}_{c}=\{(\xi, \eta) \mid 0 \leq \xi \leq 1,0 \leq \eta \leq 1\}$ is covered by the square mesh:

$$
\left\{\left(\xi_{j}, \eta_{k}\right) \mid \xi_{j}=\frac{j}{\left(J_{x}+1\right)}, \eta_{k}=\frac{k}{\left(J_{y}+1\right)} ; 0 \leq j \leq J_{x}+1,0 \leq k \leq J_{y}+1\right\} .
$$

Correspondingly, the numerical approximations to $x=x(\xi, \eta)$ and $y=y(\xi, \eta)$ are denoted by $x_{j, k}=x\left(\xi_{j}, \eta_{k}\right)$ and $y_{j, k}=y\left(\xi_{j}, \eta_{k}\right)$. As in the $1 \mathrm{D}$ case, we will derive a 


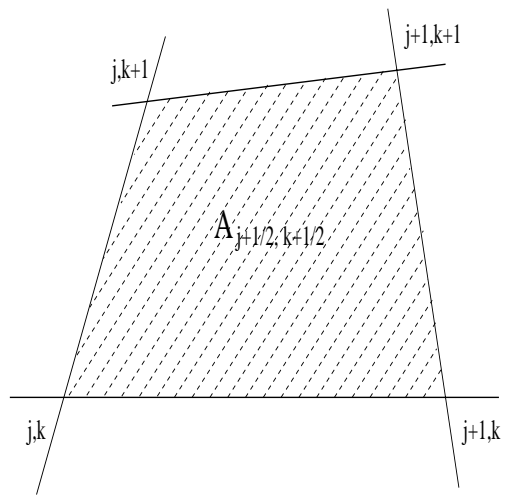

Fig. 4.1. A control volume.

conservative scheme to evaluate approximate values at new grid points. Let $A_{j+\frac{1}{2}, k+\frac{1}{2}}$ be a control volume as shown in Figure 4.1. Let $\widetilde{A}_{j+\frac{1}{2}, k+\frac{1}{2}}$ denote the quadrangle of the finite control volume with four vertices $\left(\widetilde{x}_{j+p, k+q}, \widetilde{y}_{j+p, k+q}\right), 0 \leq p, q \leq 1$, which is of setup similar to Figure 4.1.

Assume that $\widetilde{u}_{j+\frac{1}{2}, k+\frac{1}{2}}$ and $u_{j+\frac{1}{2}, k+\frac{1}{2}}$ are cell averages of $u(x, y)$ over $\widetilde{A}_{j+\frac{1}{2}, k+\frac{1}{2}}$ and $A_{j+\frac{1}{2}, k+\frac{1}{2}}$, respectively. As in the 1D case, we use the perturbation method to evaluate the numerical approximation on the resulting new grids $\left(\widetilde{x}_{j, k}, \widetilde{y}_{j, k}\right)$. If $(\widetilde{x}, \widetilde{y})=\left(x-c^{x}(x, y), y-c^{y}(x, y)\right)$, where we assume that the speeds $\left(c^{x}, c^{y}\right)$ have small amplitude, then we have

$$
\begin{aligned}
& \int_{\widetilde{A}_{j+\frac{1}{2}, k+\frac{1}{2}}} \widetilde{u}(\widetilde{x}, \widetilde{y}) d \widetilde{x} d \widetilde{y} \\
= & \int_{A_{j+\frac{1}{2}, k+\frac{1}{2}}} u\left(x-c^{x}, y-c^{y}\right) \operatorname{det}\left(\frac{\partial(\tilde{x}, \tilde{y})}{\partial(x, y)}\right) d x d y \\
\approx & \int_{A_{j+\frac{1}{2}, k+\frac{1}{2}}}\left(u(x, y)-c^{x} u_{x}-c^{y} u_{y}\right)\left(1-c_{x}^{x}-c_{y}^{y}\right) d x d y \\
\approx & \int_{A_{j+\frac{1}{2}, k+\frac{1}{2}}}\left[u(x, y)-c^{x} u_{x}-c^{y} u_{y}-c_{x}^{x} u-c_{y}^{y} u\right] d x d y \\
= & \int_{A_{j+\frac{1}{2}, k+\frac{1}{2}}}\left[u(x, y)-\left(c^{x} u\right)_{x}-\left(c^{y} u\right)_{y}\right] d x d y \\
= & \int_{A_{j+\frac{1}{2}, k+\frac{1}{2}}} u(x, y) d x d y-\left[\left(c_{n} u\right)_{j+1, k+\frac{1}{2}}+\left(c_{n} u\right)_{j, k+\frac{1}{2}}\right] \\
- & -\left[\left(c_{n} u\right)_{j+\frac{1}{2}, k+1}+\left(c_{n} u\right)_{j+\frac{1}{2}, k}\right],
\end{aligned}
$$

where we have neglected higher-order terms, $c_{n}:=c^{x} n_{x}+c^{y} n_{y}$ with $\left(n_{x}, n_{y}\right)$ the unit normal, and $\left(c_{n} u\right)_{j, k+\frac{1}{2}}$ and $\left(c_{n} u\right)_{j+\frac{1}{2}, k}$ denote the values of $c_{n} u$ at the corresponding surfaces of the control volume $A_{j+\frac{1}{2}, k+\frac{1}{2}}$. From (4.1), we obtain a conservativeinterpolation:

$$
\begin{aligned}
& \left|\widetilde{A}_{j+\frac{1}{2}, k+\frac{1}{2}}\right| \widetilde{u}_{j+\frac{1}{2}, k+\frac{1}{2}}=\left|A_{j+\frac{1}{2}, k+\frac{1}{2}}\right| u_{j+\frac{1}{2}, k+\frac{1}{2}} \\
& \quad-\left[\left(c_{n} u\right)_{j+1, k+\frac{1}{2}}+\left(c_{n} u\right)_{j, k+\frac{1}{2}}\right]-\left[\left(c_{n} u\right)_{j+\frac{1}{2}, k+1}+\left(c_{n} u\right)_{j+\frac{1}{2}, k}\right],
\end{aligned}
$$


where $|\widetilde{A}|$ and $|A|$ denote the areas of the control volumes $\widetilde{A}$ and $A$, respectively. It can be verified that the above solution-updating scheme satisfies mass-conservation:

$$
\sum_{j, k}\left|\widetilde{A}_{j+\frac{1}{2}, k+\frac{1}{2}}\right| \widetilde{u}_{j+\frac{1}{2}, k+\frac{1}{2}}=\sum_{j, k}\left|A_{j+\frac{1}{2}, k+\frac{1}{2}}\right| u_{j+\frac{1}{2}, k+\frac{1}{2}} .
$$

4.2. Solution procedure. The solution procedure of our adaptive mesh strategy for two-dimensional hyperbolic problems is almost the same as that of Algorithm 0 provided in section 3.3. Some details of the steps used for our 2D algorithm are given below.

Step i. Give an initial partition $\vec{z}_{j, k}^{[0]}=\left(x_{j, k}^{[0]}, y_{j, k}^{[0]}\right):=\left(x_{j, k}, y_{j, k}\right)$ of the physical domain $\Omega_{p}$ and a uniform (fixed) partition of the logical domain $\Omega_{c}$, and compute grid values $u_{j+\frac{1}{2}, k+\frac{1}{2}}^{[0]}$ by cell averaging the initial data $u(x, y, 0)$ over the control volume $A_{j+\frac{1}{2}, k+\frac{1}{2}}$.

Step ii. For $\nu=0,1,2, \ldots$, do the following:

(a) Move grid $\vec{z}_{j, k}^{[\nu]}=\left\{\left(x_{j, k}^{[\nu]}, y_{j, k}^{[\nu]}\right)\right\}$ to $\vec{z}_{j, k}^{[\nu+1]}=\left\{\left(x_{j, k}^{[\nu+1]}, y_{j, k}^{[\nu+1]}\right)\right\}$ by solving $\vec{z}_{\tau}=\left(\omega \vec{z}_{\xi}\right)_{\xi}+\left(\omega \vec{z}_{\eta}\right)_{\eta}$ with the conventional explicit scheme. This step can be also done by solving $\left(\omega \vec{z}_{\xi}\right)_{\xi}+\left(\omega \vec{z}_{\eta}\right)_{\eta}=0$ with the following Gauss-Seidel iteration:

$$
\begin{aligned}
& \alpha_{j+\frac{1}{2}, k}\left(\vec{z}_{j+1, k}^{[\nu]}-\vec{z}_{j, k}^{[\nu+1]}\right)-\alpha_{j-\frac{1}{2}, k}\left(\vec{z}_{j, k}^{[\nu+1]}-\vec{z}_{j-1, k}^{[\nu+1]}\right) \\
& \quad+\beta_{j, k+\frac{1}{2}}\left(\vec{z}_{j, k+1}^{[\nu]}-\vec{z}_{j, k}^{[\nu+1]}\right)-\beta_{j, k-\frac{1}{2}}\left(\vec{z}_{j, k}^{[\nu+1]}-\vec{z}_{j, k-1}^{[\nu+1]}\right)=0
\end{aligned}
$$

for $1 \leq j \leq J_{x}$ and $1 \leq k \leq J_{y}$, where

$$
\begin{aligned}
& \alpha_{j \pm \frac{1}{2}, k}=\omega\left(u_{j \pm \frac{1}{2}, k}^{[\nu]}\right)=\omega\left(\frac{1}{2}\left(u_{j \pm \frac{1}{2}, k+\frac{1}{2}}^{[\nu]}+u_{j \pm \frac{1}{2}, k-\frac{1}{2}}^{[\nu]}\right)\right), \\
& \beta_{j, k \pm \frac{1}{2}}^{[\nu]}=\omega\left(u_{j, k \pm \frac{1}{2}}^{[\nu]}\right)=\omega\left(\frac{1}{2}\left(u_{j+\frac{1}{2}, k \pm \frac{1}{2}}^{[\nu]}+u_{j-\frac{1}{2}, k \pm \frac{1}{2}}^{[\nu]}\right) .\right.
\end{aligned}
$$

(b) Compute $\left\{u_{j+\frac{1}{2}, k+\frac{1}{2}}^{[\nu+1]}\right\}$ on the new grid using the conservative-interpolation (4.2). The approximations for $c^{x}, c^{y}$, etc. are direct extensions of those defined for the $1 \mathrm{D}$ case.

(c) Repeat the updating procedure (a) and (b) for a fixed number of iterations (say, three or five) or until $\left\|\vec{z}^{[\nu+1]}-\vec{z}^{[\nu]}\right\| \leq \epsilon$.

Step iii. Evolve the underlying PDEs using 2D high-resolution finite volume methods on the mesh $\left\{\left(x_{j, k}^{[\nu+1]}, y_{j, k}^{[\nu+1]}\right)\right\}$ to obtain the numerical approximations $u_{j+\frac{1}{2}, k+\frac{1}{2}}^{n+1}$ at the time level $t_{n+1}$.

Step iv. If $t_{n+1} \leq T$, then let $u_{j+\frac{1}{2}, k+\frac{1}{2}}^{[0]}:=u_{j+\frac{1}{2}, k+\frac{1}{2}}^{n+1}$ and $\left(x_{j, k}^{[0]}, y_{j, k}^{[0]}\right):=\left(x_{j, k}^{[\nu+1]}, y_{j, k}^{[\nu+1]}\right)$, and go to Step ii.

4.3. Boundary redistribution. In many flow situations, discontinuities may initially exist in boundaries or move to boundaries at a later time. As a consequence, boundary point redistribution should be made in order to improve the quality of the solution near boundaries. A simple redistribution strategy is proposed as follows. (For convenience, our attention is restricted to the case in which the physical domain $\Omega_{p}$ is rectangular.) Assume that a new set of grid points $\left\{\tilde{x}_{j, k}, \tilde{y}_{j, k}\right\}$ is obtained in $\Omega_{p}$ by solving the moving mesh equation (4.4). Then the speeds of the internal grid point $\left(x_{j, k}, y_{j, k}\right)$ are given by

$$
\left(c^{1}, c^{2}\right)_{j, k}:=(\widetilde{x}-x, \widetilde{y}-y)_{j, k} \quad \text { for } \quad 1 \leq j \leq J_{x}, 1 \leq j \leq J_{y} .
$$


We assume that the points of the left and bottom boundaries are moving with the same speed as the tangential component of the speed for the internal points adjacent to those boundary points, namely,

$$
\begin{array}{ll}
\left(c^{1}, c^{2}\right)_{0, k}=\left(0, c_{1, k}^{2}\right), & 1 \leq j \leq J_{y}, \\
\left(c^{1}, c^{2}\right)_{j, 0}=\left(c_{j, 1}^{1}, 0\right), & 1 \leq j \leq J_{x} .
\end{array}
$$

Thus new boundary points $\left(\widetilde{x}_{0, k}, \widetilde{y}_{0, k}\right)$ and $\left(\widetilde{x}_{j, 0}, \widetilde{y}_{j, 0}\right)$ are defined by

$$
\begin{array}{ll}
(\widetilde{x}, \widetilde{y})_{0, k}=(x, y)_{0, k}+\left(c^{1}, c^{2}\right)_{0, k}, & 1 \leq k \leq J_{y}, \\
(\widetilde{x}, \widetilde{y})_{j, 0}=(x, y)_{j, 0}+\left(c^{1}, c^{2}\right)_{j, 0}, & 1 \leq j \leq J_{x} .
\end{array}
$$

The redistribution for other boundaries can be carried out in a similar way. Numerical experiments show that the above procedure for moving the boundary points is useful in improving the solution resolution.

5. Numerical experiments for $1 \mathrm{D}$ problems. In this section, we first implement our adaptive mesh methods presented in the last section for several 1D model problems. One of the main advantages of Algorithm 0 is that the solution algorithm (i.e., PDE solver) and the mesh redistribution algorithm are independent of each other. Several solution schemes, such as the MUSCL-type finite volume method (3.16)(3.18), the second-order MUSCL-type gas-kinetic approach [38], and the second-order central scheme [27], have been employed to evolve the underlying PDEs in Step 3 of Algorithm 0. The results obtained by the three methods are in good agreement.

5.1. 1D example. Three examples will be considered in this subsection. All of them have been used by several authors to test various numerical schemes.

Example 5.1. Burgers' equation. This example is the inviscid Burgers' equation

$$
u_{t}+\left(\frac{u^{2}}{2}\right)_{x}=0, \quad 0 \leq x \leq 2 \pi
$$

subject to the $2 \pi$-periodic initial data

$$
u(x, 0)=0.5+\sin (x), \quad x \in[0,2 \pi) .
$$

The solution propagates to the right, steepening until the critical time $t_{c}=1$, at which a shock forms. Figure 5.1 shows the solutions at $t=2$, when the shock is well developed. Also shown in Figure 5.1 is the trajectory of the grid points up to $t=2$, obtained with $J=30$ and $J=50$. The ability of the adaptive mesh method to capture and follow the moving shock is clearly demonstrated in this figure. Some details in this example are the following: the monitor function used in the computation is $\omega=\sqrt{1+0.2\left|u_{\xi}\right|^{2}}$; the number of Gauss-Seidel iterations used is 5; the scheme for evolving Burgers' equation is a (formally) second-order MUSCL finite volume scheme (with the Lax-Friedrichs flux) together with a second-order RungeKutta discretization; the CFL number used is 0.3 .

In Table 5.1, $L^{1}$-error and convergence rate are listed for $t=0.9$ and $t=0.999$. It is observed that a second-order rate of convergence can be obtained for the adaptive mesh method.

Example 5.2. Nonconvex conservation laws. Here we apply the adaptive mesh algorithm to the Riemann problem of a scalar hyperbolic conservation law with a nonlinear nonconvex flux:

$$
u_{t}+f(u)_{x}=0, \quad f(u)=\frac{1}{4}\left(u^{2}-1\right)\left(u^{2}-4\right) .
$$


$\mathrm{u}$
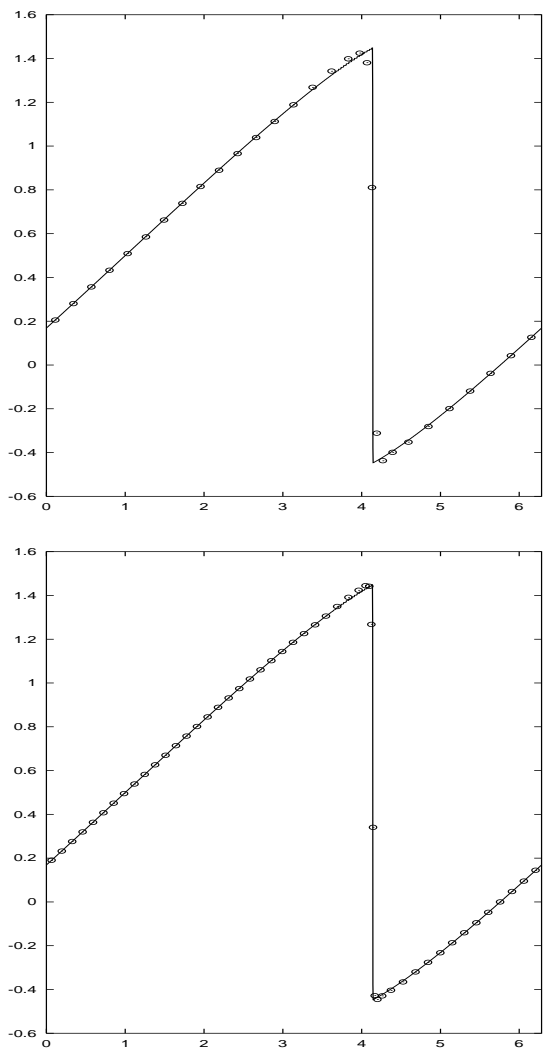

t
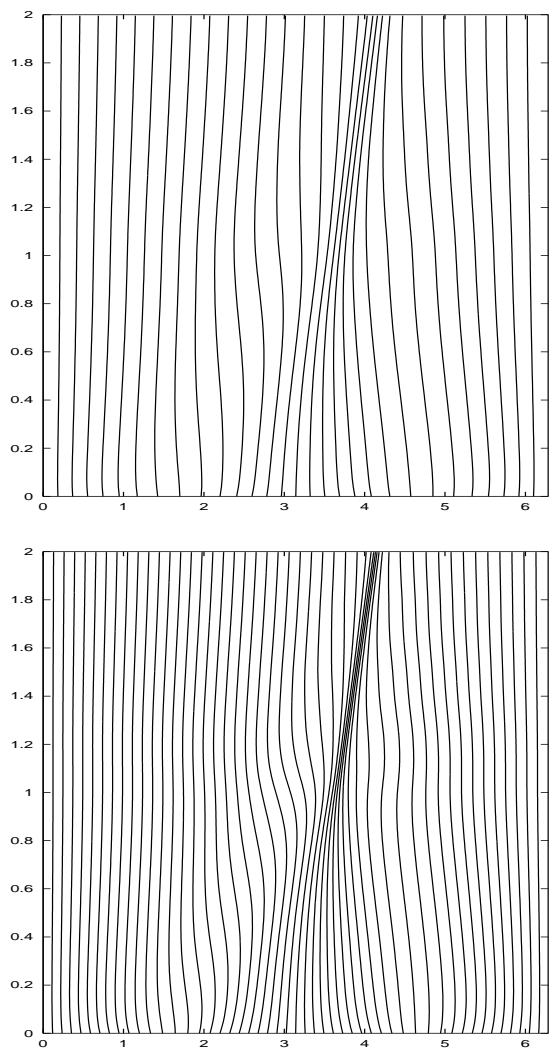

Fig. 5.1. Example 5.1. Left: numerical ("o") and exact solutions (solid line) at $t=2$. Right: trajectory of the mesh for $0 \leq t \leq 2$. Top: $J=30$; and bottom: $J=50$.

TABLE 5.1

Example 5.1: $L^{1}$-error and convergence order at $t=0.9$ and $t=0.999$.

\begin{tabular}{|c|c|c|c|c|}
\hline $\mathrm{J}$ & 40 & 80 & 160 & 320 \\
\hline$t=0.9$ & $4.73 \mathrm{e}-2 \quad(-)$ & $1.48 \mathrm{e}-2 \quad(1.68)$ & $3.76 \mathrm{e}-3 \quad(1.98)$ & $7.90 \mathrm{e}-4 \quad(2.25)$ \\
\hline$t=0.999$ & $5.84 \mathrm{e}-2 \quad(-)$ & $1.85 \mathrm{e}-2 \quad(1.67)$ & $5.23 \mathrm{e}-3 \quad(1.82)$ & $1.33 \mathrm{e}-3 \quad(1.98)$ \\
\hline
\end{tabular}

The initial data are $u(x, 0)=-2 \operatorname{sign}(x)$.

The problem was also considered in [17]. In contrast with Burgers' equation, the flux function for this problem is nonconvex, which leads to difficulties with some numerical schemes and so serves as a good test problem. The numerical solution at $t=1.2$ is shown for an adaptive mesh in Figure 5.2, with $J=30$ and 50. Some details in this example are the following: the monitor function used in the computation is $\omega=$ $\sqrt{1+\left|u_{\xi}\right|^{2}}$; the number of Gauss-Seidel iterations is 5 ; the scheme for evolving (5.2) is a (formally) second-order MUSCL finite volume scheme (with the Lax-Friedrichs flux) together with a second-order Runge-Kutta discretization. It is seen that the numerical solution gives sharp shock profiles.

Example 5.3. Euler equations of gas dynamics. In this example, we test our 

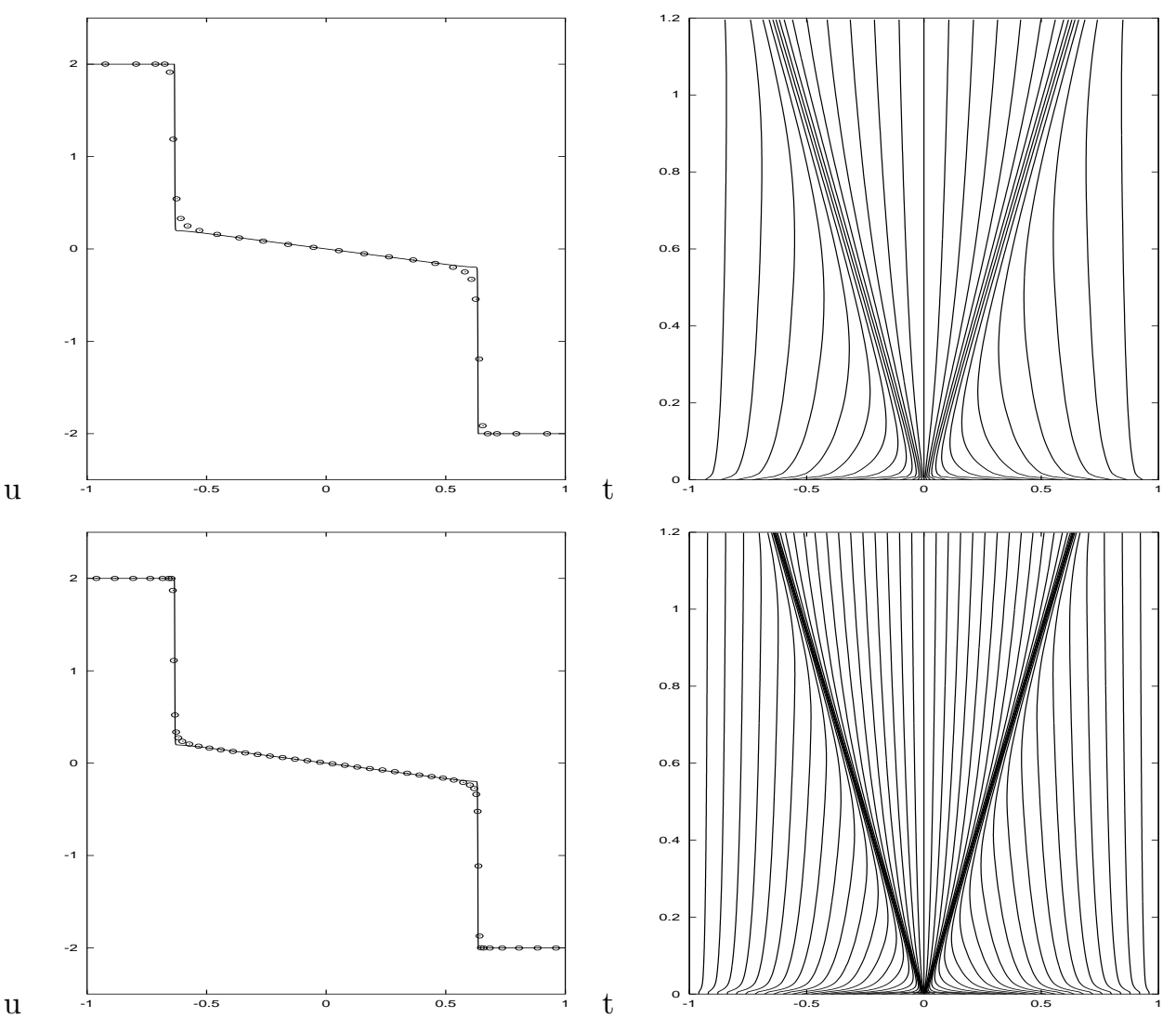

FiG. 5.2. Example 5.2. Left: numerical ("o") and exact solutions (solid line) at $t=1.2$. Right: trajectory of the mesh for $0 \leq t \leq 1.2$. Top: $J=30$; and bottom: $J=50$.

adaptive mesh algorithm with the one-dimensional Euler equations of gas dynamics,

$$
\left[\begin{array}{c}
\rho \\
\rho u \\
E
\end{array}\right]_{t}+\left[\begin{array}{c}
\rho u \\
\rho u^{2}+p \\
u(E+p)
\end{array}\right]_{x}=0
$$

where $\rho, u, p$, and $E$ are density, velocity, pressure, and total energy, respectively. The above system is closed by the equation of state, $p=(\gamma-1)\left(E-\rho u^{2} / 2\right)$. The initial data are chosen as

$$
(\rho, \rho u, E)= \begin{cases}(1,0,2.5) & \text { if } x<0.5 \\ (0.125,0,0.25) & \text { if } x>0.5 .\end{cases}
$$

This is a well known test problem proposed by Sod [32]. The monitor function employed for this computation is $G=\omega I$ with

$$
\omega=\sqrt{1+\alpha_{1}\left(\frac{u_{\xi}}{\max _{\xi}\left|u_{\xi}\right|}\right)^{2}+\alpha_{2}\left(\frac{s_{\xi}}{\max _{\xi}\left|s_{\xi}\right|}\right)^{2}}
$$

where $s=p / \rho^{\gamma}$, and the parameters $\alpha_{i}(i=1,2)$ are some nonnegative constants. The above monitor function was suggested by Stockie, Mackenzie, and Russell [33], who also discussed several other choices for the monitor function. The numerical results are obtained with $J=100, \alpha_{1}=20, \alpha_{2}=100$ and are presented in Figure 5.3. 
(a)

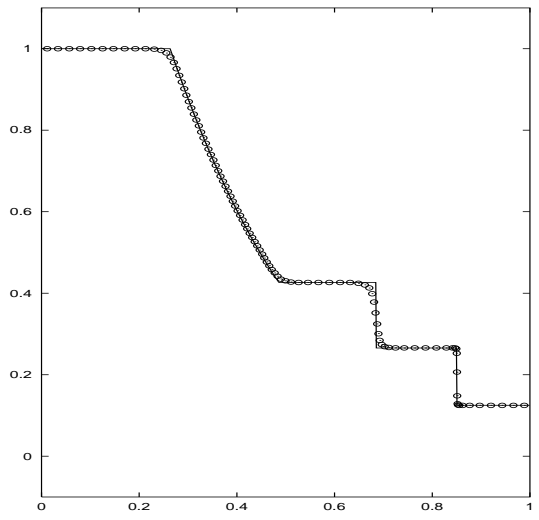

(c)

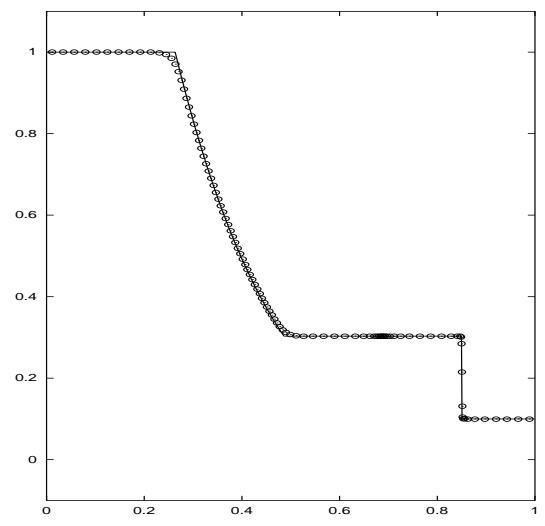

(b)

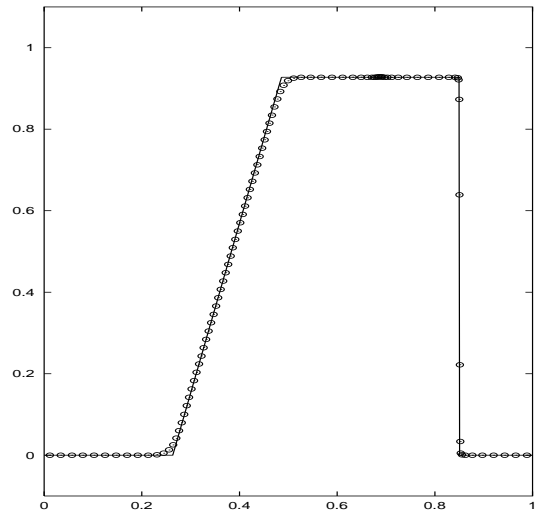

(d)

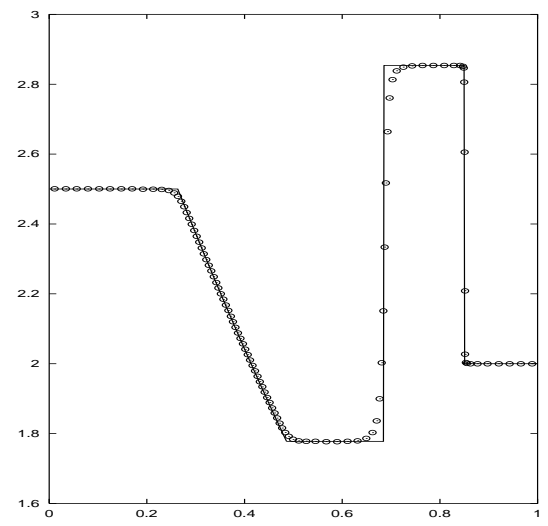

FIG. 5.3. Example 5.3: adaptive mesh solution at $t=0.2$. (a) density, (b) velocity, (c) pressure, and (d) internal energy. "o" and solid lines denote numerical and exact solutions, respectively.

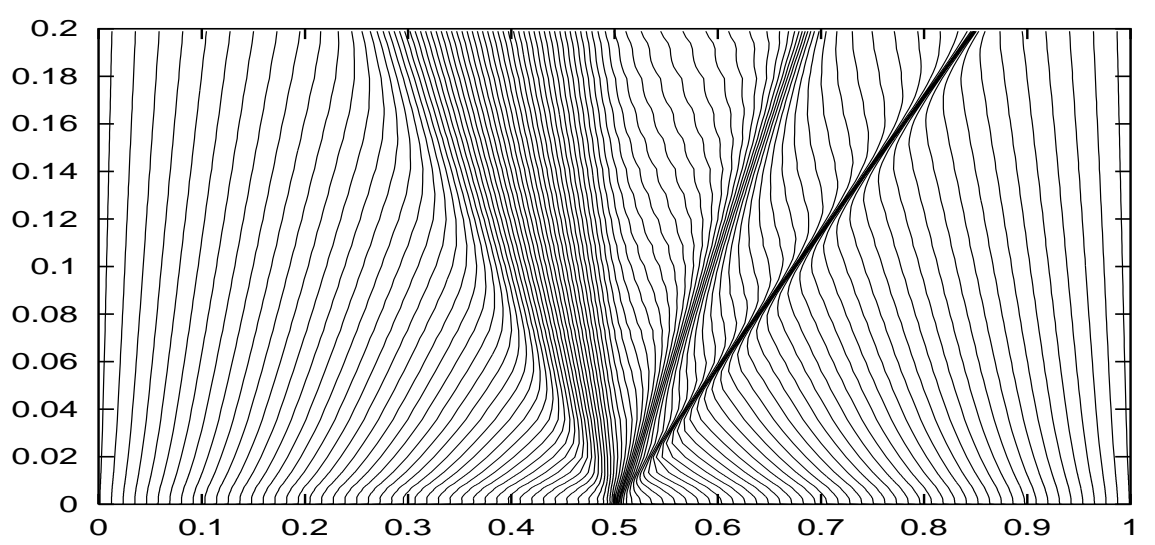

FIG. 5.4. Example 5.3: trajectory of the grid points.

It is found that the contact and shock discontinuities are well resolved, although quite a number of grid points are also moved to the rarefaction wave region. This can also be observed from the mesh contour plotted in Figure 5.4. 
6. Numerical experiment for $2 \mathrm{D}$ problems. In this section, we will test our adaptive mesh algorithm presented in section 3.3 for some $2 \mathrm{D}$ problems, including 2D Riemann problems, a double-Mach reflection problem, and flow past a circular cylinder.

6.1. 2D grid generation. We begin by testing Step 2 of Algorithm 0, i.e., testing the mesh distribution part with given functions.

Example 6.1. $2 D$ grid generation. We consider grid generation in the physical domain $[-1,1] \times[-1,1]$ for the following functions:

$$
\begin{aligned}
& u(x, y)=\exp \left(-8\left(4 x^{2}+9 y^{2}-1\right)^{2}\right), \\
& u(x, y)=\exp \left(-100\left(y-x^{2}+0.5\right)^{2}\right), \\
& u(x, y)=50 \exp \left(-2500\left(x^{2}+y^{2}\right)\right), \\
& u(x, y)= \begin{cases}1 & \text { if }|x| \leq|y|, \\
0 & \text { otherwise. }\end{cases}
\end{aligned}
$$

The monitor function is taken as $G=\omega I$ with $\omega=\sqrt{1+\alpha u^{2}}$, with $\alpha=100$. Grid generations based on the above functions have been investigated by many authors; see e.g., $[7,21,28]$. Our results plotted in Figure 6.1 can be favorably compared with published results. Our results indicate that Step 2 of Algorithm 0 for two dimensions performs well for functions with large gradients or singularities.

(a)

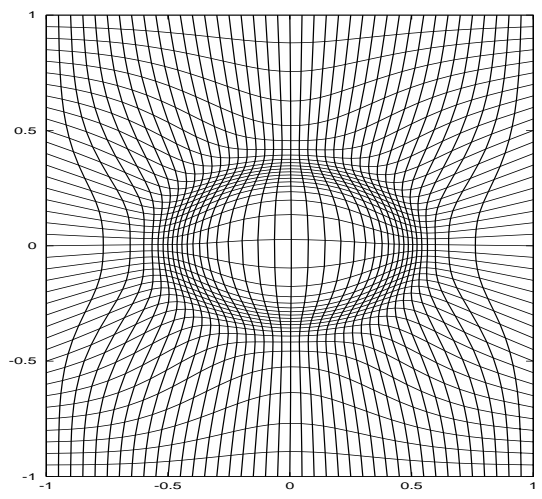

(c)

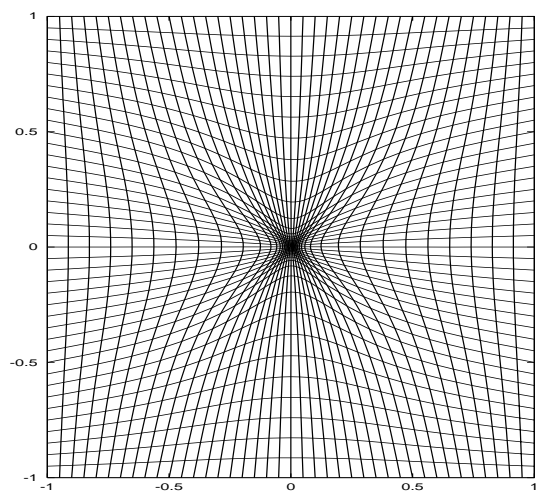

(b)

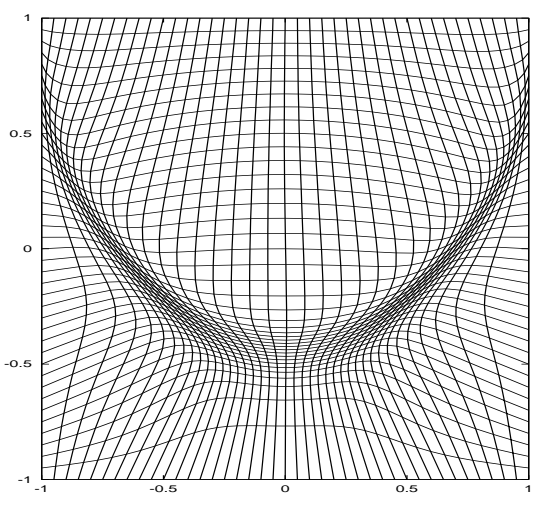

(d)

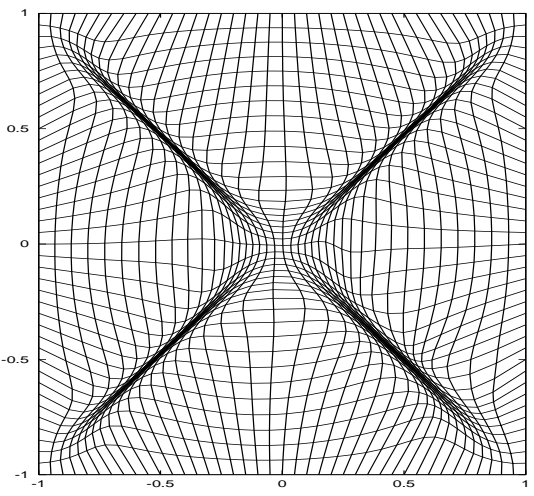

FIG. 6.1. Example 6.1: the adaptive meshes for (a) function (6.1), (b) function (6.2), (c) function (6.3), and (d) function (6.4). 
6.2. 2D examples for Euler equations of gas dynamics. In this subsection we consider some well known test examples in two dimensions, including three Riemann problems and a double-Mach reflection problem.

Example 6.2. $2 D$ Riemann problem I: Shock waves. Two-dimensional Euler equations of gas dynamics can be written as

$$
\left[\begin{array}{c}
\rho \\
\rho u \\
\rho v \\
E
\end{array}\right]_{t}+\left[\begin{array}{c}
\rho u \\
\rho u^{2}+p \\
\rho u v \\
u(E+p)
\end{array}\right]_{x}+\left[\begin{array}{c}
\rho v \\
\rho u v \\
\rho v^{2}+p \\
v(E+p)
\end{array}\right]_{y}=0
$$

where $\rho,(u, v), p$, and $E$ are the density, velocity, pressure, and total energy, respectively. For an ideal gas, the equation of state, $p=(\gamma-1)\left(E-\rho\left(u^{2}+v^{2}\right) / 2\right)$, is provided. The initial data are chosen as

$$
(\rho, u, v, p)= \begin{cases}(1.1,0.0,0.0,1.1) & \text { if } x>0.5, \quad y>0.5 \\ (0.5065,0.8939,0.0,0.35) & \text { if } x<0.5, \quad y>0.5 \\ (1.1,0.8939,0.8939,1.1) & \text { if } x<0.5, \quad y<0.5 \\ (0.5065,0.0,0.8939,0.35) & \text { if } x>0.5, \quad y<0.5\end{cases}
$$

which corresponds to the case of left forward shock, right backward shock, upper backward shock, and lower forward shock. We refer the readers to $[19,30]$ for details.

In [19], Lax and Liu computed 2D Riemann problems with various initial data using positive schemes. The problem considered here corresponds to Configuration 4 discussed in their paper. We use our adaptive mesh algorithm with $\left(J_{x}, J_{y}\right)=(50,50)$ and $\left(J_{x}, J_{y}\right)=(100,100)$ to compute this Riemann problem and display the mesh and density at $t=0.25$ in Figure 6.2. It is found that our results with $J_{x}=J_{y}=100$ give sharper shock resolution than that of the positive schemes with $\left(J_{x}, J_{y}\right)=(400,400)$ (see $[19$, p. 333]). The monitor function used in this computation is $G=\omega I$, with $\omega=\sqrt{1+2\left(\rho_{\xi}^{2}+\rho_{\eta}^{2}\right)} x$.

Example 6.3. $2 D$ Riemann problem II: Contact discontinuities. We reconsider Configurations 6 and 7 in Lax and Liu's paper [19], whose solutions contain contact discontinuities. The first configuration has initial data

$$
(\rho, u, v, p)= \begin{cases}(1,0.75,-0.5,1) & \text { if } x>0.5, \quad y>0.5 \\ (2,0.75,0.5,1) & \text { if } x<0.5, \quad y>0.5 \\ (1,-0.75,0.5,1) & \text { if } x<0.5, \quad y<0.5 \\ (3,-0.75,-0.5,1) & \text { if } x>0.5, \quad y<0.5\end{cases}
$$

and the second configuration has initial data

$$
(\rho, u, v, p)= \begin{cases}(1,0.1,0.1,1) & \text { if } x>0.5, \quad y>0.5 \\ (0.5197,-0.6259,0.1,0.4) & \text { if } x<0.5, \quad y>0.5 \\ (0.8,0.1,0.1,0.4) & \text { if } x<0.5, \quad y<0.5 \\ (0.5197,0.1,-0.6259,0.4) & \text { if } x>0.5, \quad y<0.5\end{cases}
$$

The adaptive mesh results for Configuration 6 at $t=0.3$ and for Configuration 7 at $t=0.25$ are displayed in Figure 6.3. The number of grid points are $\left(J_{x}, J_{y}\right)=$ $(100,100)$. It can be observed that the adaptive mesh results with $J_{x}=J_{y}=100$ for Configuration 7 are comparable with those obtained by using the positive schemes with $J_{x}=J_{y}=400$ (see [19, p. 334]). However, this seems not to be the case for the results for Configuration 6 . Although the resolution can be improved by using more 

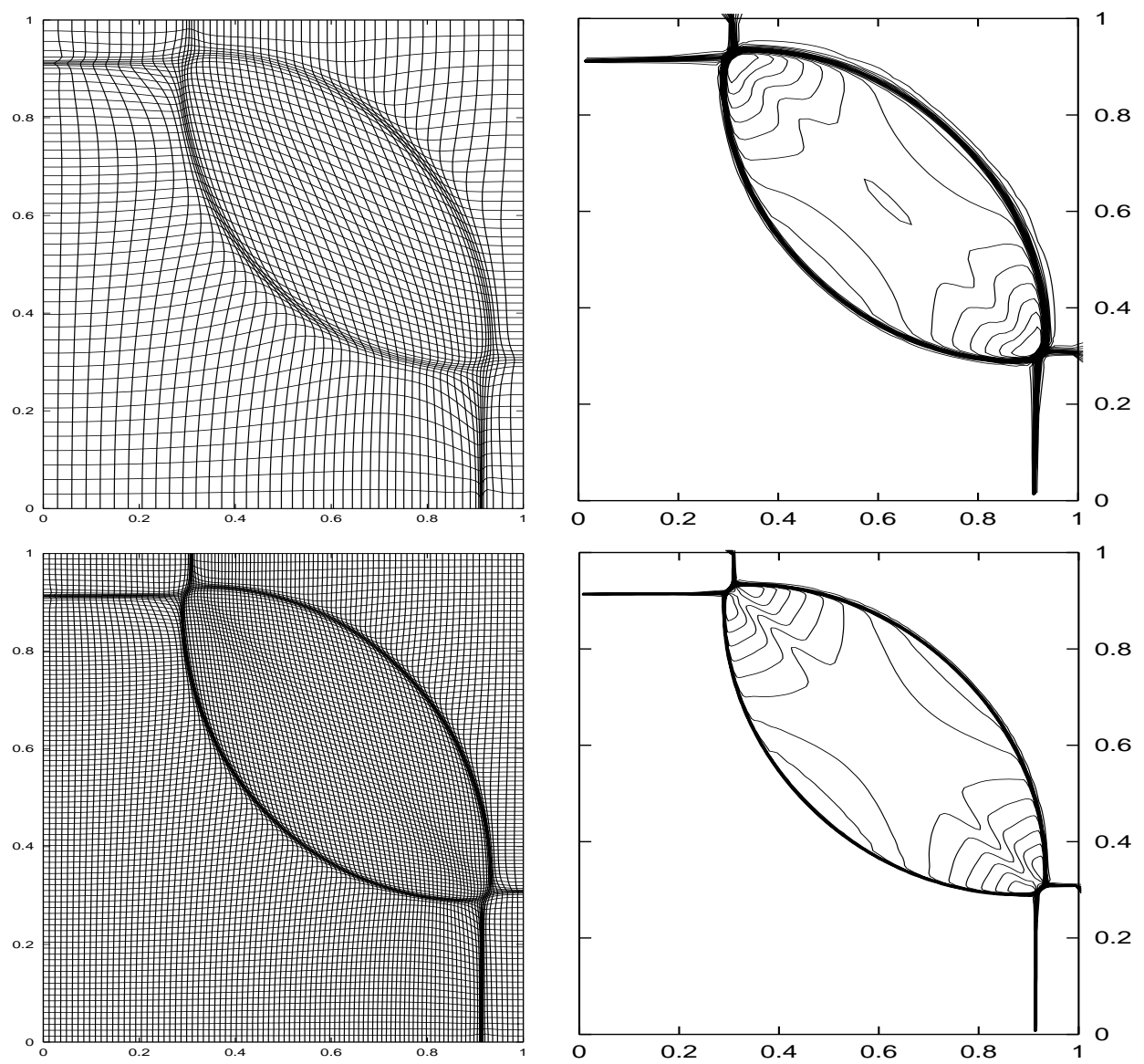

FIG. 6.2. Example 6.2. The contours of the mesh (left) and the density (right). Top: $J_{x}=$ $J_{y}=50$; and bottom: $J_{x}=J_{y}=100.30$ equally spaced contour lines are used for the density.

grid points, it is quite clear from this computation and Example 6.2 that the treatment of contact discontinuities is less effective than the treatment of shocks. It is expected that the monitor functions used in this paper are suitable for shock discontinuities but may be less appropriate for contact discontinuities. Therefore, it requires further investigation to obtain more effective monitor functions for contact discontinuities.

For this computation, the monitor function can be chosen as $G=\omega I$, with $\omega=$ $\sqrt{1+\alpha\left(\rho_{\xi}^{2}+\rho_{\eta}^{2}\right)}$. It is found that in both cases if the parameter $\alpha$ is chosen in the range $[0.1,1]$, then the efficiency and effectiveness of the adaptive mesh approach seem satisfactory. The results in Figure 6.3 are obtained using $\alpha=0.1$ (for Configuration 6) and $\alpha=0.9$ (for Configuration 7).

Example 6.4. The double-Mach reflection problem. This problem was studied extensively in Woodward and Colella [37] and later by many others. We use exactly the same setup as in [37], i.e., the same initial and boundary conditions and same solution domain $\Omega_{p}=[0,4] \times[0,1]$. Initially a right-moving Mach 10 shock is positioned at $x=\frac{1}{6}, y=0$ and makes a $60^{\circ}$ angle with the $x$-axis. More precisely, the initial data are

$$
U= \begin{cases}U_{L} & \text { for } y \geq h(x, 0) \\ U_{R} & \text { otherwise }\end{cases}
$$



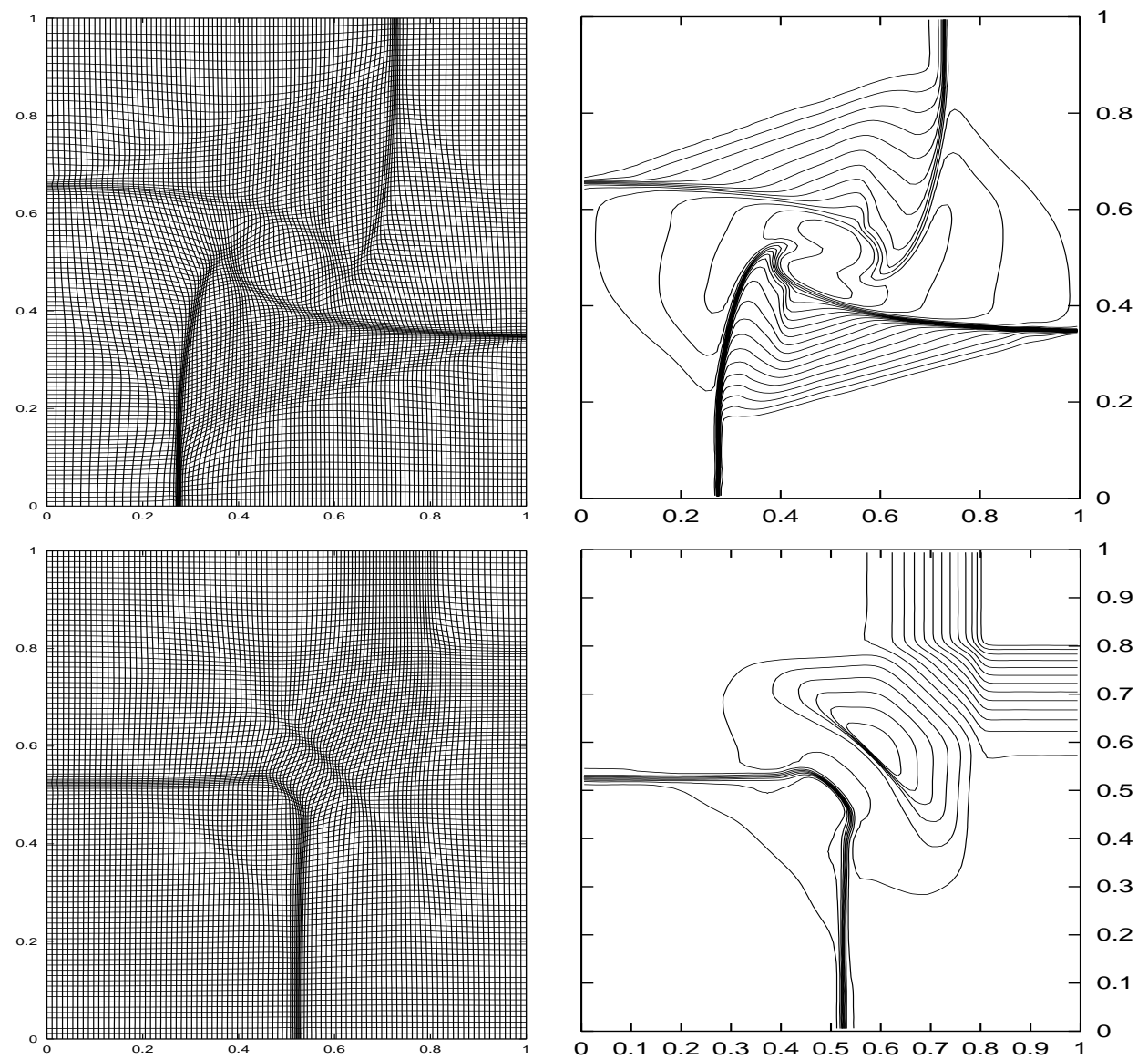

FIG. 6.3. Adaptive mesh results for Example 6.3 with $100 \times 100$ grid points. Top: Configuration 6; bottom: Configuration 7. Left: the adaptive mesh; Right: density. 19 equally spaced contour lines are used for the density.

where the state on the left, the state on the right, and the shock strength are, respectively,

$$
\begin{aligned}
& U_{L}=(8,57.1597,-33.0012,563.544)^{T}, \\
& U_{R}=(1.4,0.00 .0,2.5)^{T}, \quad h(x, t)=\sqrt{3}(x-1 / 6)-20 t .
\end{aligned}
$$

As in [37], only the results in $[0,3] \times[0,1]$ are displayed. In Figure 6.4 , the adaptive meshes with $\left(J_{x}, J_{y}\right)=(80,20),(160,40)$, and $(320,80)$ are displayed, while the corresponding contours of density are displayed in Figure 6.5. By comparing the density plots, it is found that the adaptive computation results with $\left(J_{x}, J_{y}\right)=(320,80)$ have similar resolution to the results obtained by the second-order discontinuous Galerkin method with $\left(J_{x}, J_{y}\right)=(960,240)$ (see $[10$, p. 214]) and by the second-order central scheme with $\left(J_{x}, J_{y}\right)=(960,240)$ (see $[10$, p. 67]). Moreover, the adaptive results with $\left(J_{x}, J_{y}\right)=(160,40)$ have slightly better resolution than the results of fifth-order weighted ENO and the fourth-order ENO with $480 \times 119$ grids [10, p. 406]. Of course, this is not too surprising, since these published results are computed using uniform meshes. 

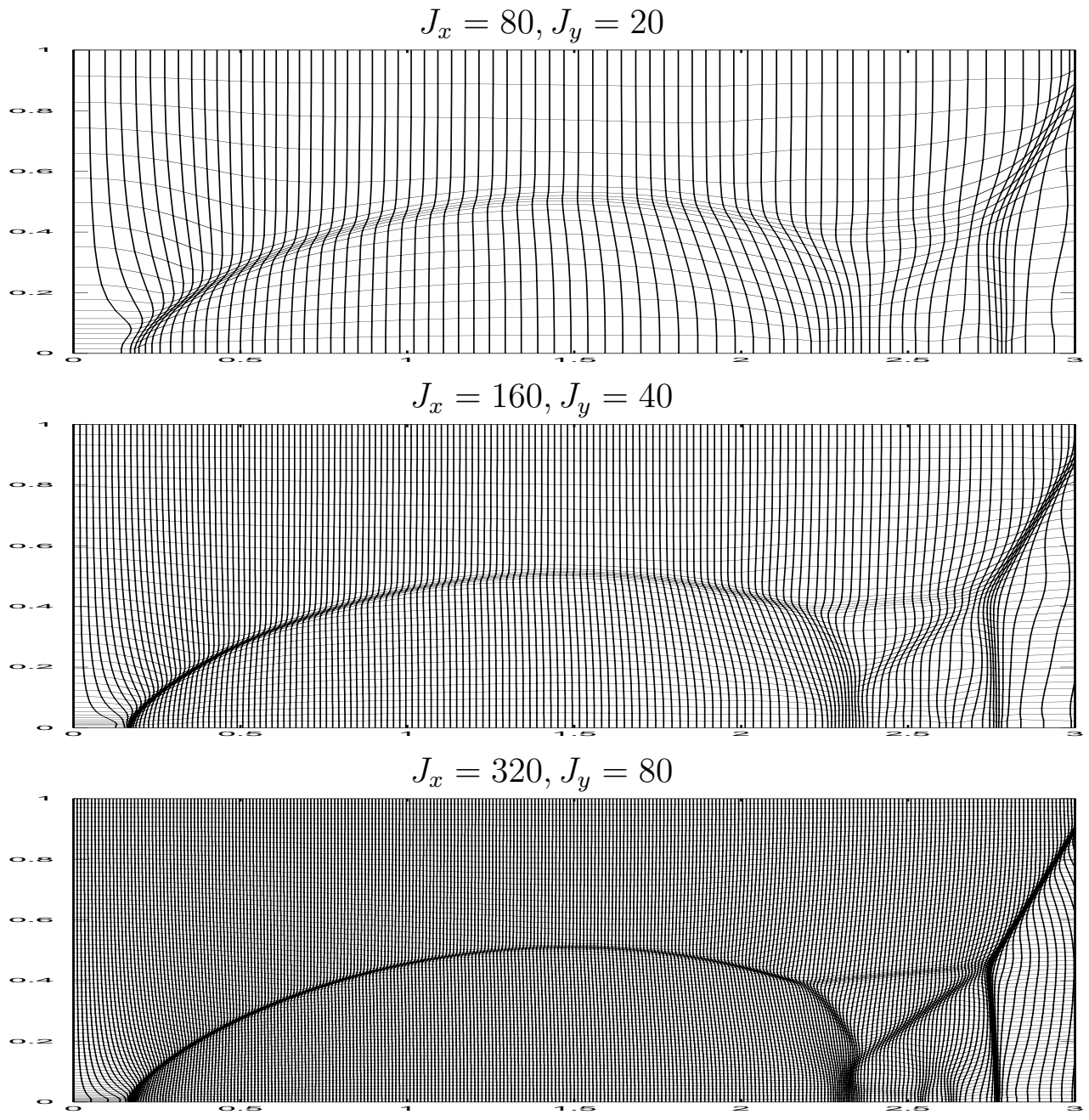

FIG. 6.4. $2 D$ double-Mach reflection at $t=0.2$ : the contours of meshes. From top to bottom: $\left(J_{x}, J_{y}\right)=(80,20),(160,40)$, and $(320,80)$.

We also show a blow up portion around the double-Mach region in Figure 6.6. In our computations, we used $640 \times 160$ and $960 \times 240$ grid points. The corresponding mesh contours in the blow up region are shown in Figure 6.7. The smallest $\Delta x$ and $\Delta y$ in these runs are listed in Tables 6.1 and 6.2. It is seen that ratios between the largest and smallest mesh sizes in the adaptive grids are quite large $(\geq 20)$, which is a desired feature of the adaptive grid methods. The fine details of the complicated structure in this region were previously obtained by Cockburn and Shu [9], who used high-order discontinuous Galerkin (RKDG) methods with $960 \times 240$ and $1920 \times 480$ grid points. Although the moving mesh algorithm gives a good resolution in this blow up portion, it is observed that, even with approximately the same number of grid points $(960 \times 240)$, the third-order RKDG results [10, p. 216] have a slightly better resolution of the complex structure. The monitor function used for this example is taken as $G=\omega I$, with $\omega=\sqrt{1+0.125\left(\rho_{\xi}^{2}+\rho_{\eta}^{2}\right)}$. 


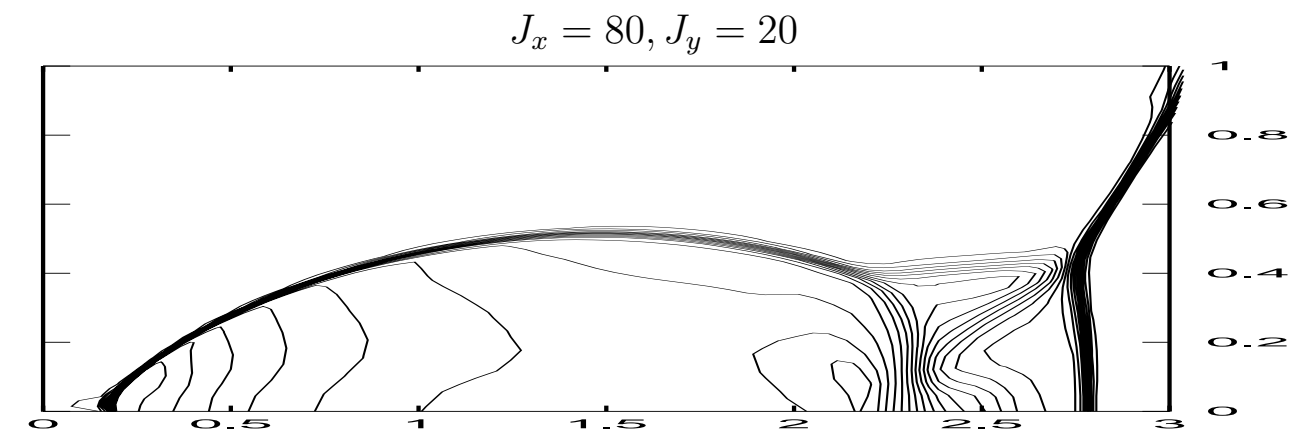

$$
J_{x}=160, J_{y}=40
$$

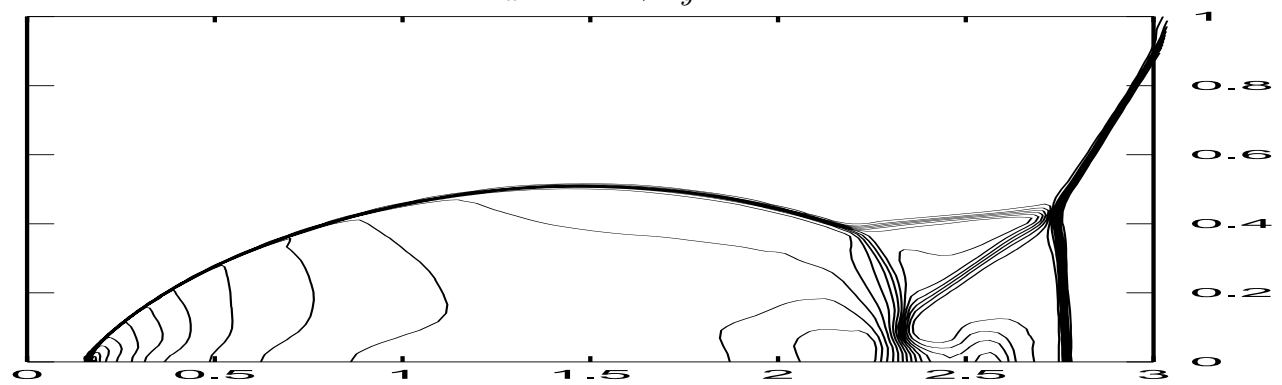

$J_{x}=320, J_{y}=80$

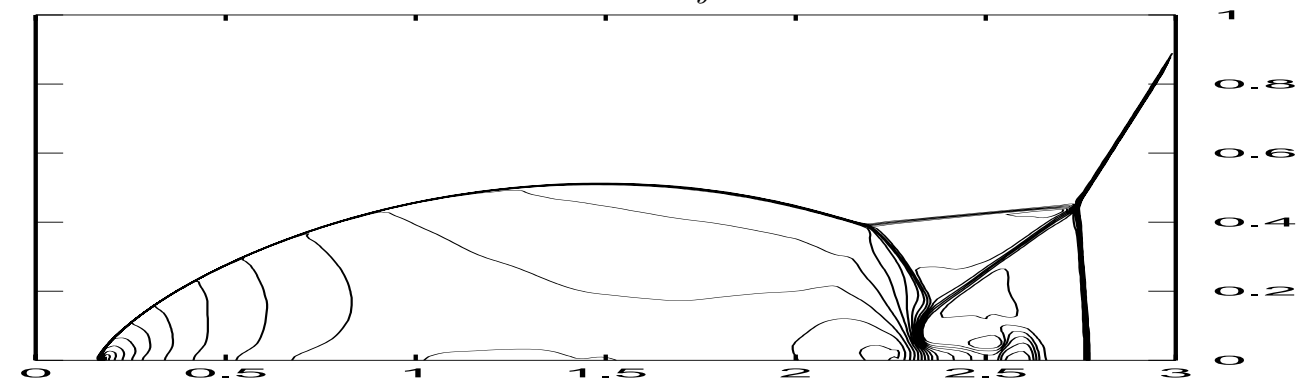

FIG. 6.5. $2 D$ double-Mach reflection at $t=0.2$ : the contours of density. From top to bottom: $\left(J_{x}, J_{y}\right)=(80,20),(160,40)$, and $(320,80) .30$ equally spaced contour lines are used.

6.3. Example of a nonconvex physical domain. So far, the numerical examples in two dimensions have been restricted to rectangular domains. In the final example, we consider a test problem whose domain is not even convex. In this case, as long as the domain can be smoothly transformed to a rectangle, the adaptive mesh algorithm can be handily applied.

Example 6.5. Flow past a cylinder. This example is concerned with the supersonic flow past a cylinder with unit radius, which is positioned at the origin on an $x-y$ plane. The problem is initialized by a Mach 3 free-stream moving toward the cylinder from the left. Since the physical domain $\Omega_{p}$ is nonconvex, we first transform $\Omega_{p}$ to a square domain $\widehat{\Omega}_{c}=[0,1] \times[0,1]$ by using the following mapping:

$$
\begin{aligned}
& x=-\left(R_{x}-\left(R_{x}-1\right) \widehat{x}\right) \cos (\theta(2 \widehat{y}-1)), \\
& y=\left(R_{y}-\left(R_{y}-1\right) \widehat{x}\right) \sin (\theta(2 \widehat{y}-1)),
\end{aligned}
$$




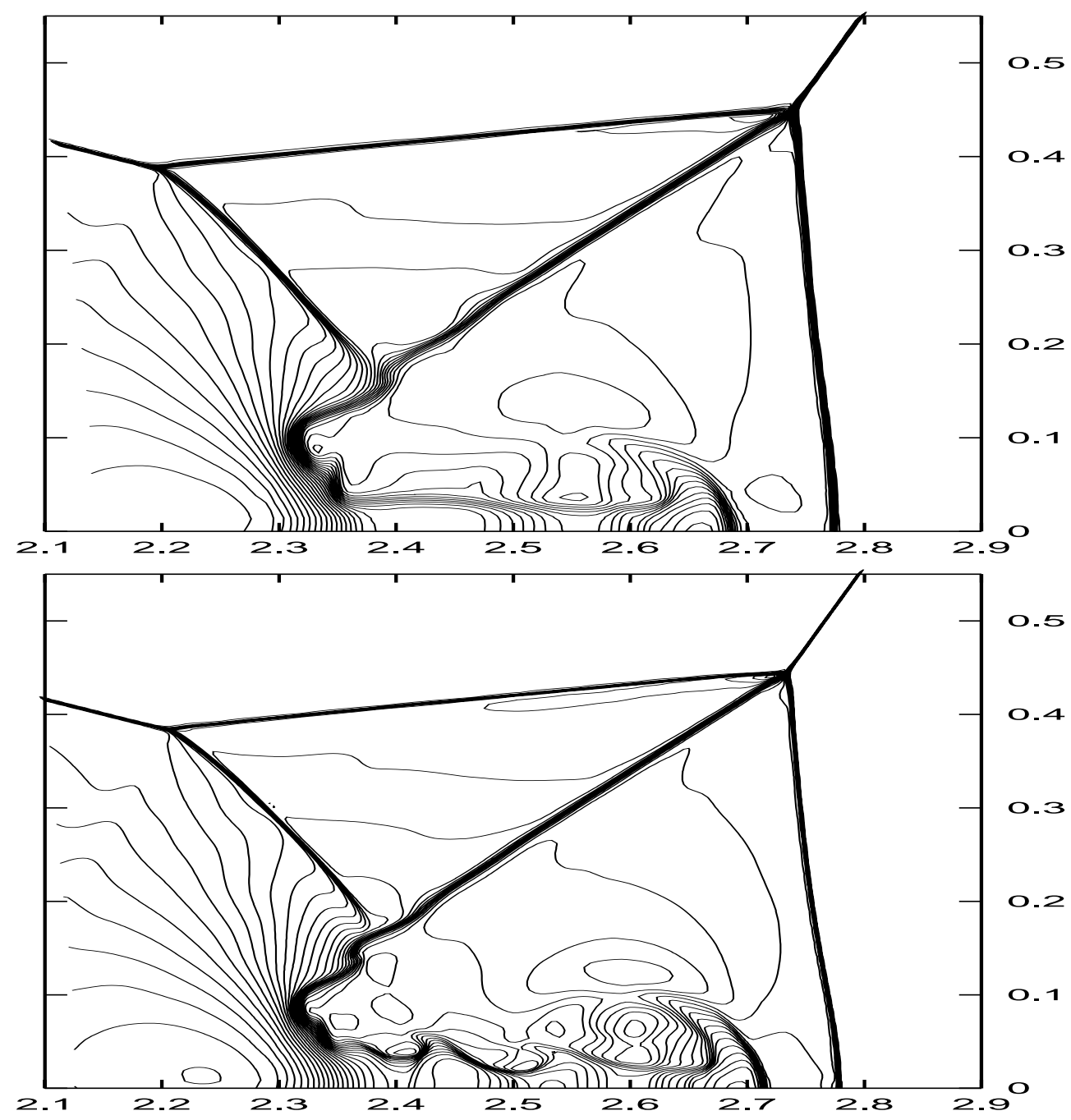

FIG. 6.6. Double-Mach reflection problem: density $\rho$ in blowup region around the double-Mach stems. Top: $\left(J_{x}, J_{y}\right)=(640,160)$; bottom: $\left(J_{x}, J_{y}\right)=(960,240) .45$ equally spaced contour lines are used.

with $R_{x}=3, R_{y}=5$, and $\theta=5 \pi / 12$. A reflective boundary condition is imposed at the surface of the cylinder, i.e., $\hat{x}=1$; inflow boundary condition is applied at $\hat{x}=0$; and outflow boundary conditions are applied at $\hat{y}=0$ and 1 . We then solve the problem in $\widehat{\Omega}_{c}$ using the adaptive mesh algorithm, with a logical domain $\Omega_{c}$ as before. This procedure will lead to numerical solution in $\widehat{\Omega}_{c}$, and the mapping (6.6) finally gives the numerical approximation in the physical domain $\Omega_{p}$.

We present an illustration of the mesh in the physical space and the pressure contour in Figure 6.8, by using $30 \times 40,60 \times 80$, and $120 \times 160$ grid points. The monitor function used for this example is taken as $G=\omega I$ with $\omega=\sqrt{1+0.125\left(\rho_{\xi}^{2}+\rho_{\eta}^{2}\right)}$. As can be seen from these figures, the advantages of the adaptive mesh methods are quite obvious. The shock location computed by our adaptive mesh algorithm is approximately 0.703 (the distance between the shock curve and the surface of the cylinder), which is in good agreement with the experimental results reported in [4]. 

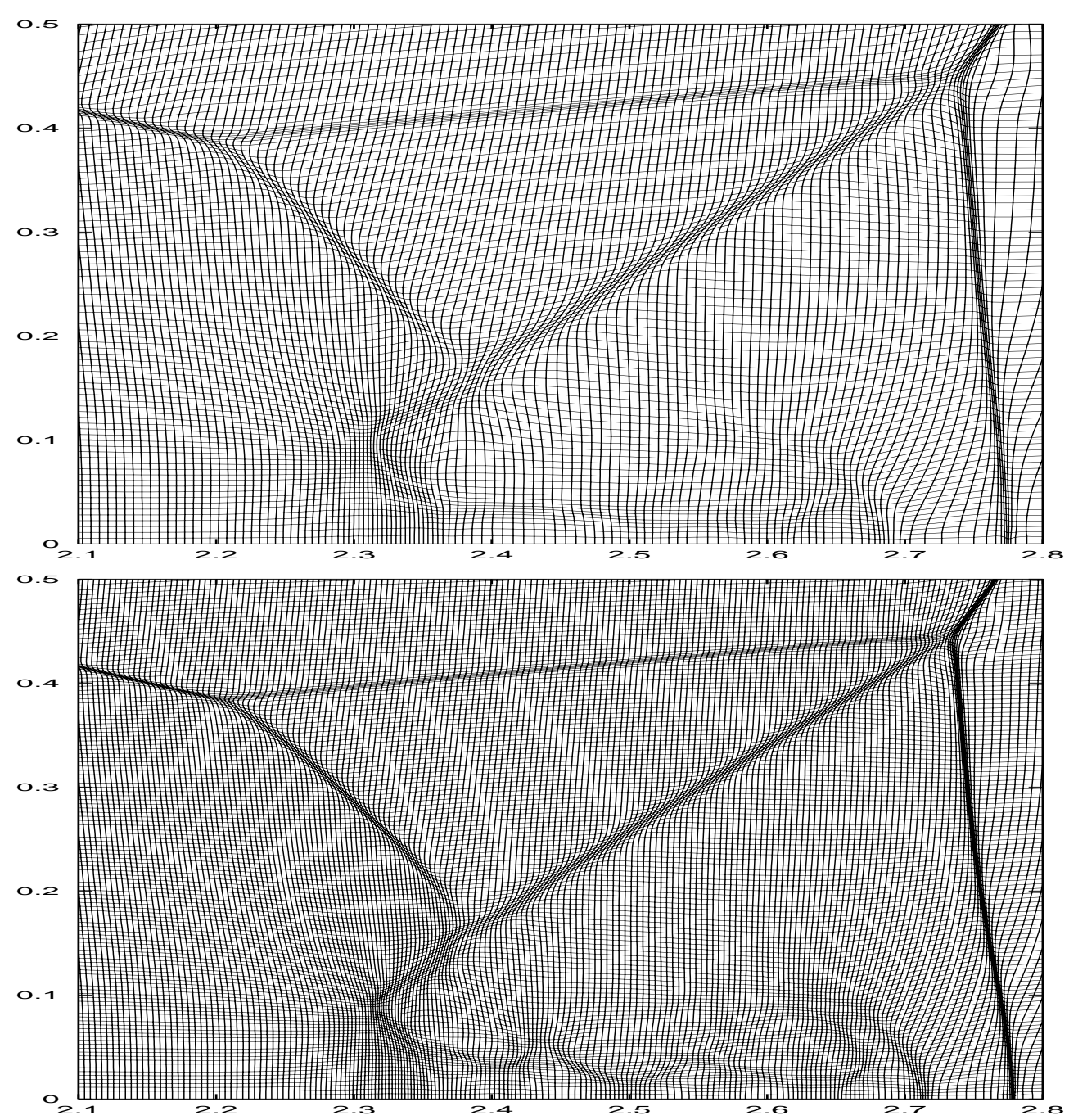

FIG. 6.7. Double-Mach reflection problem: the adaptive mesh in blowup region around the double-Mach stems. Top: $\left(J_{x}, J_{y}\right)=(640,160)$; bottom: $\left(J_{x}, J_{y}\right)=(960,240)$.

TABLE 6.1

The smallest mesh size for the double-Mach reflection problem with $640 \times 160$ grid points.

\begin{tabular}{|c||c|c|c|}
\hline & $\min \{\Delta x\}$ & $\max \{\Delta x\}$ & $\max \{\Delta x\} / \min \{\Delta x\}$ \\
\hline$\Delta x$ & $6.5 \mathrm{e}-04$ & $2.0 \mathrm{e}-02$ & 30.8 \\
\hline$\Delta y$ & $4.8 \mathrm{e}-04$ & $1.0 \mathrm{e}-02$ & 20.8 \\
\hline$\sqrt{\Delta x^{2}+\Delta y^{2}}$ & $8.2 \mathrm{e}-04$ & $2.0 \mathrm{e}-02$ & 24.4 \\
\hline
\end{tabular}

Acknowledgments. The authors thank Professors Chi-Wang Shu and Eitan Tadmor for numerous discussions during the preparation of this work. We also thank the referees for many helpful suggestions. 
TABLE 6.2

The smallest mesh sizes for the double-Mach reflection problem with $960 \times 240$ grid points.

\begin{tabular}{|c||c|c|c|}
\hline & $\min \{\Delta x\}$ & $\max \{\Delta x\}$ & $\max \{\Delta x\} / \min \{\Delta x\}$ \\
\hline$\Delta x$ & $4.3 \mathrm{e}-04$ & $1.1 \mathrm{e}-02$ & 25.6 \\
\hline$\Delta y$ & $3.1 \mathrm{e}-04$ & $5.9 \mathrm{e}-03$ & 19.0 \\
\hline$\sqrt{\Delta x^{2}+\Delta y^{2}}$ & $5.3 \mathrm{e}-04$ & $1.2 \mathrm{e}-02$ & 22.6 \\
\hline
\end{tabular}
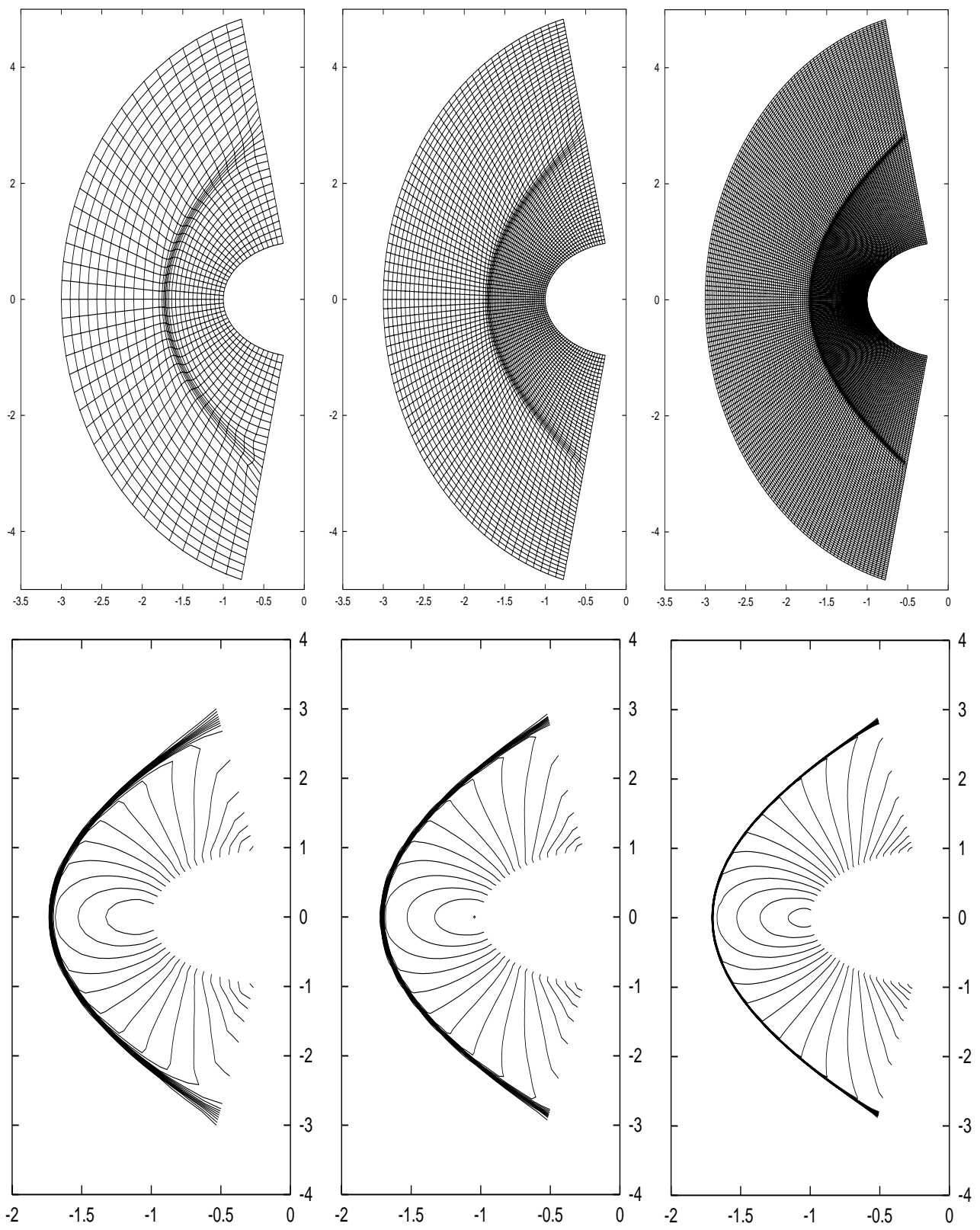

FIG. 6.8. Example 6.5. Top: adaptive mesh; bottom: pressure. From left to right: $30 \times 40$, $60 \times 80$, and $120 \times 160$ grid points. 20 equally spaced contour lines are used for the pressure. 


\section{REFERENCES}

[1] B. N. AzARENoK, Variational barrier method of adaptive grid generation in hyperbolic problems of gas dynamics, SIAM J. Numer. Anal., 40 (2002), pp. 651-682.

[2] B. N. Azarenok and S. A. Ivanenko, Application of adaptive grids in numerical analysis of time-dependent problems in gas dynamics, Comput. Math. Math. Phys., 40 (2000), pp. $1330-1349$.

[3] B. N. Azarenok, S. A. Ivanenko, and T. Tang, Adaptive mesh redistribution method based on Godunov's scheme, Comm. Math. Sci., 1 (2003), pp. 152-179.

[4] O. M. Belotserkovskil, Computation of flows around the circular cylinder with detached shock waves, Comput. Math., 3 (1958), pp. 149-185 (in Russian).

[5] J. U. BrackBill, An adaptive grid with directional control, J. Comput. Phys., 108 (1993), pp. $38-50$.

[6] J. U. Brackbill and J. S. Saltzman, Adaptive zoning for singular problems in two dimensions, J. Comput. Phys., 46 (1982), pp. 342-368.

[7] W. M. CaO, W. Z. Huang, and R. D. Russell, An r-adaptive finite element method based upon moving mesh PDEs, J. Comput. Phys., 149 (1999), pp. 221-244.

[8] H. D. Ceniceros And T. Y. Hou, An efficient dynamically adaptive mesh for potentially singular solutions, J. Comput. Phys., 172 (2001), pp. 609-639.

[9] B. Cockburn and C.-W. Shu, The Runge-Kutta discontinuous Galerkin method for conservation laws V: Multidimensional systems, J. Comput. Phys., 141 (1998), pp. 199-224.

[10] B. Cockburn, C. Johnson, C.-W. Shu, and E. Tadmor, Advanced Numerical Approximation of Nonlinear Hyperbolic Equations, papers from the C.I.M.E. Summer School in Centraro, Italy, 1997, A. Quarteroni, ed., Lecture Notes in Math. 1697, Springer-Verlag, Berlin, 1998.

[11] S. F. Davis And J. E. Flaherty, An adaptive finite element method for initial-boundary value problems for partial differential equations, SIAM J. Sci. Statist. Comput., 3 (1982), pp. 6-27.

[12] A. S. Dvinsky, Adaptive grid generation from harmonic maps on Riemannian manifolds, J. Comput. Phys., 95 (1991), pp. 450-476.

[13] R. FAZIO And R. LeVeque, Moving-mesh methods for one-dimensional hyperbolic problems using CLAWPACK, Comp. Math. Appl., to appear.

[14] A. Harten and J. M. Hyman, Self-adjusting grid methods for one-dimensional hyperbolic conservation laws, J. Comput. Phys., 50 (1983), pp. 235-269.

[15] K. H. Karlsen, K.-A. Lie, and N. H. Risebro, A front tracking method for conservation laws with boundary conditions, in Hyperboilic Problems: Theory, Numerics, Applications, M. Fey and R. Jeltsch, eds., Internat. Ser. Numer. Math. 129, Birkhäuser Verlag, 1999, pp. 493-502.

[16] S. Jin AND Z. P. XIN, The relaxation schemes for systems of conservation laws in arbitrary space dimensions, Comm. Pure Appl. Math., 48 (1995), pp. 235-276.

[17] A. KURGANOV AND E. TADMOR, New high-resolution central schemes for nonlinear conservation laws and convective-diffusion equations, J. Comput. Phys., 160 (2000), pp. 214-282.

[18] P. D. LAX AND X. D. LiU, Positive schemes for solving multi-dimensional hyperbolic systems of conservation laws, J. Comput. Fluid Dynam., 5 (1996), pp. 133-156.

[19] P. D. LaX AND X.-D. LiU, Solutions of two-dimensional Riemann problems of gas dynamics by positive schemes, SIAM J. Sci. Comput., 19 (1998), pp. 319-340.

[20] R. J. LEVEQUE, High-resolution finite volume methods on arbitrary grids via wave propagation, J. Comput. Phys., 78 (1988), pp. 36-63.

[21] R. Li, T. TANG, AND P. W. Zhang, Moving mesh methods in multiple dimensions based on harmonic maps, J. Comput. Phys., 170 (2001), pp. 562-588.

[22] R. Li, T. TANG, AND P. W. Zhang, A moving mesh finite element algorithm for singular problems in two and three space dimensions, J. Comput. Phys., 177 (2002), pp. 365-393.

[23] S. Li And L. Petzold, Moving mesh methods with upwinding schemes for time-dependent PDEs, J. Comput. Phys., 131 (1997), pp. 368-377.

[24] F. Liu, S. Ji, AND G. LiaO, An adaptive grid method and its application to steady Euler flow calculations, SIAM J. Sci. Comput., 20 (1998), pp. 811-825.

[25] K. Miller and R. N. Miller, Moving finite elements. I, SIAM J. Numer Anal., 18 (1981), pp. 1019-1032.

[26] R. NATANLini, Convergence to equilibrium for the relaxation approximations of conservation laws, Comm. Pure Appl. Math., 49 (1996), pp. 795-823.

[27] H. Nessyahu and E. TADmor, Non-oscillatory central differencing for hyperbolic conservation laws, J. Comput. Phys., 87 (1990), pp. 408-463.

[28] W. Q. REN AND X. P. WANG, An iterative grid redistribution method for singular problems in 
multiple dimensions, J. Comput. Phys., 159 (2000), pp. 246-273.

[29] K. Saleri and S. Steinberg, Flux-corrected transport in a moving grid, J. Comput. Phys., 111 (1994), pp. 24-32.

[30] C. W. Schulz-Rinne, J. P. Collins, And H. M. Glaz, Numerical solution of the Riemann problem for two-dimensional gas dynamics, SIAM J. Sci. Comput., 14 (1993), pp. 13941414.

[31] C.-W. Shu And S. Osher, Efficient implementation of essentially non-oscillatory shockcapturing schemes II, J. Comput. Phys., 83 (1989), pp. 32-78.

[32] G. A. SoD, A survey of finite difference methods for systems of nonlinear hyperbolic conservation laws, J. Comput. Phys., 27 (1978), pp. 1-31.

[33] J. M. Stockie, J. A. Mackenzie, and R. D. Russell, A moving mesh method for onedimensional hyperbolic conservation laws, SIAM J. Sci. Comput., 22 (2001), pp. 17911813.

[34] E. TAdmor and T. TAng, Pointwise error estimates for relaxation approximations to conservation laws, SIAM J. Math. Anal., 32 (2000), pp. 870-886.

[35] H. Z. TANG AND H.-M. WU, Kinetic flux vector splitting for radiation hydrodynamical equations, Comput. \& Fluids, 29 (2000), pp. 917-933.

[36] A. Winslow, Numerical solution of the quasi-linear Poisson equation, J. Comput. Phys., 1 (1967), pp. 149-172.

[37] P. Woodward and P. Colella, The numerical simulation of two dimensional fluid flow with strong shocks, J. Comput. Phys., 54 (1984), pp. 115-173.

[38] K. Xu, Gas-Kinetic Schemes for Unsteady Compressible Flow Simulations, lecture notes from the von Karman Institute for Fluid Dynamics Lecture Series 1998-03, Rhode-Saint-Genèse, Belgium, 1998.

[39] H. YANG, A local extrapolation method for hyperbolic conservation laws I: The ENO underlying schemes, J. Sci. Comput., 15 (2000), pp. 231-264. 